\title{
Molecular and functional signatures in a novel Alzheimer's disease mouse model assessed by quantitative proteomics
}

Dong Kyu Kim ${ }^{1 \dagger}$, Joonho Park ${ }^{2 \dagger}$, Dohyun Han ${ }^{3 \dagger}$, Jinhee Yang ${ }^{1}$, Ahbin Kim ${ }^{1}$, Jongmin Woo ${ }^{1}$, Youngsoo Kim² and Inhee Mook-Jung ${ }^{1,4^{*}}$

\begin{abstract}
Background: Alzheimer's disease (AD), the most common neurodegenerative disorder, is characterized by the deposition of extracellular amyloid plaques and intracellular neurofibrillary tangles. To understand the pathological mechanisms underlying $A D$, developing animal models that completely encompass the main features of $A D$ pathologies is indispensable. Although mouse models that display pathological hallmarks of AD (amyloid plaques, neurofibrillary tangles, or both) have been developed and investigated, a systematic approach for understanding the molecular characteristics of AD mouse models is lacking.

Methods: To elucidate the mechanisms underlying the contribution of amyloid beta (A $\beta$ ) and tau in AD pathogenesis, we herein generated a novel animal model of AD, namely the AD-like pathology with amyloid and neurofibrillary tangles $\left(A D L P^{A P T}\right.$ ) mice. The ADLPAPT mice carry three human transgenes, including amyloid precursor protein, presenilin-1, and tau, with six mutations. To characterize the molecular and functional signatures of AD in ADLPAT mice, we analyzed the hippocampal proteome and performed comparisons with individual-pathology transgenic mice (i.e., amyloid or neurofibrillary tangles) and wild-type mice using quantitative proteomics with 10-plex tandem mass tag.

Results: The ADLP ${ }^{A P T}$ mice exhibited accelerated neurofibrillary tangle formation in addition to amyloid plaques, neuronal loss in the CA1 area, and memory deficit at an early age. In addition, our proteomic analysis identified nearly 10,000 protein groups, which enabled the identification of hundreds of differentially expressed proteins (DEPS) in ADLP ${ }^{\text {APT }}$ mice. Bioinformatics analysis of DEPs revealed that ADLP ${ }^{A P T}$ mice experienced age-dependent active immune responses and synaptic dysfunctions.

Conclusions: Our study is the first to compare and describe the proteomic characteristics in amyloid and neurofibrillary tangle pathologies using isobaric label-based quantitative proteomics. Furthermore, we analyzed the hippocampal proteome of the newly developed $A D L P^{A P T}$ model mice to investigate how both $A \beta$ and tau pathologies regulate the hippocampal proteome. Because the ADLPAPT mouse model recapitulates the main features of AD pathogenesis, the proteomic data derived from its hippocampus has significant utility as a novel resource for the research on the Aß-tau axis and pathophysiological changes in vivo.
\end{abstract}

Keywords: Alzheimer's disease, Animal disease model, Aß, Tau, Quantitative proteomics, 10-plex tandem mass tag

\footnotetext{
*Correspondence: biolab@snu.ac.kr; inhee@snu.ac.kr

${ }^{\dagger}$ Equal contributors

${ }^{2}$ Interdisciplinary Program in Bioengineering, College of Engineering, Seoul

National University, 1 Gwanak-ro, Seoul 151-742, South Korea

'Department of Biomedical Sciences, Seoul National University, College of

Medicine, 103 Daehak-ro, Seoul 110-799, South Korea

Full list of author information is available at the end of the article
} 


\section{Background}

Alzheimer's disease (AD) is the most common neurodegenerative disorder and is characterized by extracellular amyloid plaque deposition, intracellular neurofibrillary tangle (NFT) development, and memory impairments $[1,2]$. Although the disease has been extensively studied over the past several decades, the exact mechanisms and pathological causes of AD remain unclear. Genetically modified mouse models that recapitulate the major features of $\mathrm{AD}$ pathologies are invaluable in determining the underlying disease mechanisms and evaluating new therapeutic approaches. Few transgenic mouse models, such as the triple transgenic mice, 3xTg-AD model, have been introduced to develop the concomitant manifestation of both amyloid plaques and NFT formation [3]. Because this model showed no neuronal cell death in the hippocampus, the development of a new mouse model that fully mimics $A D$ pathologies is still needed.

One of the most widely used models in AD research is the 5xFAD (Tg6799) model, which contains five familial AD mutations within the APP and PSEN1 genes. The $5 x F A D$ model is considered to be an effective AD model because of the rapid progression of the amyloid pathology [4]. The JNPL3 mouse model, which expresses P301Lmutant human tau, has been widely used to examine intraneuronal NFTs [5]. To recapitulate the main features of $\mathrm{AD}$ pathogenesis, we herein developed a new mouse model that carries six mutations within transgenes encoding human amyloid precursor protein (APP), presenilin-1 (PSEN1), and tau. The resulting transgenic mouse model, Alzheimer's disease-like pathology with APP, PSEN1, and $M A P T$ transgenes (ADLP ${ }^{\mathrm{APT}}$ ), exhibited $\mathrm{A} \beta$ accumulation, NFTs, early neuronal loss in the brain, and subsequent memory impairments. The pathological phenotypes of $A D L P^{A P T}$ mice, which feature both $\mathrm{A} \beta$ deposition and NFTs, were compared with those of $\operatorname{ADLP}^{\mathrm{APP} / \mathrm{PS} 1}$ (A $\beta$ deposition, no NFTs) and ADLP ${ }^{\text {Tau }}$ (no A $\beta$ deposition, NFTs) mice of the same genetic background. Understanding the interplay between $A \beta$ accumulation and NFTs is imperative for elucidating the pathogenesis of AD. Thus, the ADLP ${ }^{\mathrm{APT}}$ mouse model, which shows both robust amyloid and NFT pathologies, should be an excellent model for examining the A $\beta$-tau axis in vivo.

The hippocampus is known to play an important role in memory formation [6]. Thus, understanding the pathological status of the hippocampus under $\mathrm{AD}$ is crucial for studying the mechanisms of AD-related memory impairments. Although some mechanisms that contribute to AD pathogenesis have been uncovered by studies on individual genes or proteins, systematic analysis of the pathological changes of the hippocampus is lacking. Mass spectrometry (MS)-based proteomics is expected to be an appropriate tool for systematic analysis [7, 8]. Although MS-based proteomics have been limited because of the incomplete coverage of the proteome, recent technological advances allowed researchers to study comprehensively up to 10,000 proteins from a single cell line [9]. However, this level of coverage requires extensive pre-fractionation, large samples, and several months of instrument time [10]. In addition, the reliable MS-based quantitation under several perturbation states requires the use of biological and technical replicates, thus increasing the complexity of MS experiments. Importantly, these shortcomings can be overcome by an isobaric labeling strategy, such as the application of tandem mass tags (TMTs) [11]. The recent expansion of multiplexing capacity up to 10 samples per MS injection has markedly increased the scope of quantitative proteomics [12]. In TMT experiments, protein quantification is accomplished by comparing the intensities of reporter ions produced during MS/MS [11]. Since this approach enables sensitive and precise protein quantification, many research groups have successfully used TMTbased strategies [13-16].

With the aid of 10-plex TMT quantification strategy combined with high-resolution MS, we constructed a comprehensive proteome map of the newly developed mouse models. We have successfully discovered nearly 10,000 proteins and quantified 7000 proteins from the hippocampus of wild type, ADLP ${ }^{\mathrm{APP} / \mathrm{PS} 1}, \mathrm{ADLP}^{\mathrm{Tau}}$, and $\mathrm{ADLP} \mathrm{APT}^{\mathrm{APT}}$ mice. The protein abundances of ADLP ${ }^{\mathrm{APT}}$ mice were compared with those of other single transgenic mice to discover differentially expressed proteins and characterize functional signatures of ADLP ${ }^{\mathrm{APT}}$ mice via bioinformatics analysis. Furthermore, our network analysis could suggest the presence of interacting proteins that connect between amyloid and NFT pathologies. In conclusion, new ADLP ${ }^{\mathrm{APT}}$ mice and their hippocampal proteome dataset may help to offer a novel insight of pathogenesis of $\mathrm{AD}$ in further studies targeting the concurrent molecular network of amyloid and NFT pathologies.

\section{Methods \\ Experimental design}

The aim of this study was to construct a mouse model of Alzheimer's disease that carries mutant human genes and to introduce its molecular and functional characteristics. The protein expressions of newly constructed mouse models were assessed by quantitative proteomics combined with LC-MS/MS and TMT isobaric labeling. A total of 36 hippocampi samples were used in the proteomic experiments. ( 4 mouse types * 3 age-points * biological triplicates), which were randomly divided into four 10-plex TMT experimental sets. All samples were analyzed twice via MS. Three to twelve mice were sacrificed accordingly to the type of biochemical experiment, which includes western blotting and immunostaining. Behavioral tests of the $\mathrm{AD}$ model mice were performed by investigators in blind with respect to genotypes. No data were excluded. 


\section{Reagents and materials}

Tandem mass tag (TMT) 10-plex isobaric reagents, bicinchoninic acid (BCA) assay kit - reducing agent compatible, tris (2-carboxyethyl) phosphine (TCEP), and LC/ MS-grade solvents such as acetone, acetonitrile (ACN), and water were purchased from Thermo Fisher Scientific (Waltham, MA). Other reagents and materials were purchased from the following companies: Dithiothreitol (DTT) and urea from AMRESCO (Solon, OH), Sodium dodecyl sulfate (SDS), Trizma base from USB (Cleveland, $\mathrm{OH})$ and sequencing-grade modified trypsin from Promega Corporation (Madison, WI), POROS20 R2 bead from Applied Biosystems (Foster City, CA). High-purity (>97\%) mass spectrometry (MS) grade ovalbumin from Protea (Morgantown, WV), HLB OASIS column from Waters (Milford, MA). All other reagents, unless noted, were purchased from Sigma-Aldrich (St. Louis, MO).

\section{Transgenic mice}

5XFAD mice (Tg6799; Jackson Laboratory, Stock\#006554) express both mutant human APP with the Swedish, Florida, and London mutations and mutant human PSEN1 with the M146 L and L286 V mutations under the murine Thy1 promoter. JNPL3 mice (TauP301L-JNPL3; Taconic, Stock\#2508 homozygote) carry mutant human tau with the P301L mutation under the murine prion protein promoter. Due to the mixed genetic background of JNPL3 mice, JNPL3 mice were backcrossed with B6SJL (C57BL/6 $\mathrm{X}$ SJL) mice. The resulting JNPL3 mice on the B6SJL genetic background were crossed with 5XFAD mice to create a novel animal model, ADLP animal model. This carries the three human mutant genes and its corresponding mutations mentioned before. Only female mice were used for pathological characterization due to earlier signs of aggravated pathologies and memory deficit than male mice.

\section{Immunohistochemistry (IHC)}

Mice were anesthetized and perfused with $4 \%$ paraformaldehyde (PFA) solution in phosphate-buffered saline (PBS). The brain tissues were fixed with $4 \%$ PFA for $20 \mathrm{~h}$ at $4{ }^{\circ} \mathrm{C}$, incubated in $30 \%$ sucrose (wt/vol) for $72 \mathrm{~h}$ and then frozen. The frozen brains were cut into $30 \mu \mathrm{m}$ coronal sections using a Leica CM 1850 Cryostat. Brain slices were washed with PBS and then incubated in $70 \%$ formic acid in PBS for 20 min to perform antigen retrieval when amyloid plaques and NFTs were stained. Brain slices were permeabilized and blocked with blocking solution $(0.3 \%$ Triton $\mathrm{X}-100,5 \%$ horse serum, and $0.05 \%$ BSA solution) for $1 \mathrm{~h}$ at $20{ }^{\circ} \mathrm{C}$ prior to incubating it with primary antibodies overnight. Amyloid plaques were stained with the biotin-4G8 antibody (1:700, COVANCE), followed by the streptavidin-488-conjugated secondary antibody (Invitrogen). Hyperphosphorylated tau was examined by using the AT8 (1:300, Thermo
Scientific) and AT180 (1:300, Thermo Scientific) antibody, which recognize the Ser202/Thr205 epitopes and Thr231 of human tau, followed by biotinylated antimouse IgG (Vector Laboratories) and streptavidin-594conjugated secondary antibody (Invitrogen). To visualize astrocytes and microglia, anti-GFAP (1:1000, Invitrogen) for astrocytes and anti-Iba-1 (1:500, Wako) antibodies were used respectively. Hippocampal neurons of the CA1 layer were visualized by staining with anti-NeuN (1:1000, Millipore) antibody. Stained brain slices were incubated with goat anti-rat Alexa 488, donkey antirabbit Alexa 488, and donkey anti-mouse Alexa 647 antibody (1:500, Life Technologies) for $1 \mathrm{~h}$ at $20^{\circ} \mathrm{C}$. Images were obtained using LSM 700 (Carl Zeiss). At least six serial sections of each sample were imaged to consider the volume of cells in brain slices. When the number of neuronal cells in CA1 layer stained by anti-NeuN antibody were counted, one middle region of the hippocampus tissue was imaged to avoid an overlap of CA1 pyramidal neurons. All images were quantified using ImageJ software (NIH).

\section{Sarkosyl-insoluble tau fractionation}

One side of the hippocampus was homogenized in 8 volumes of Tris buffer solution (TBS) including phosphatase inhibitors and protease by tissue grinder [TBS solution; $25 \mathrm{mM}$ Tris/HCl, pH 7.4, $150 \mathrm{mM} \mathrm{NaCl}, 1 \mathrm{mM}$ EDTA, $1 \mathrm{mM}$ EGTA, phenylmethylsulfonyl fluoride (PMSF), protease 1, and 2]. The homogenates were centrifuged at $14,000 \mathrm{x}$ g at $4{ }^{\circ} \mathrm{C}$ for $15 \mathrm{~min}$. The supernatant was collected for further fractionation. The supernatant was incubated with N-Lauroylsarcosine sodium salt solution $20 \%$ solution (1\% final concentration) at $37{ }^{\circ} \mathrm{C}$ rotator for $1 \mathrm{~h}$ and then ultracentrifuged at $150,000 \mathrm{x}$ g at $25^{\circ} \mathrm{C}$ for $1 \mathrm{~h}$. The resulting sarkosyl-insoluble pellets were resuspended in TBS solution for washing pellets. The mixture was concentrated by ultracentrifuge again at $150,000 \times \mathrm{g}$ at $25^{\circ} \mathrm{C}$ for $1 \mathrm{~h}$. The resulting pellets containing tau aggregates were suspended with $5 x$ Sample buffer (Serva Blue G) and heated at $70{ }^{\circ} \mathrm{C}$ for $10 \mathrm{~min}$ to prevent further aggregation.

\section{Behavioral test}

For Y-maze test, after introduction to the middle of the maze, the mouse was allowed to freely explore new environments for $8 \mathrm{~min}$. Spatial memory function was measured as the percent of spontaneous alteration [17]. The number of total arm entries and the sequence of the Ymaze arm into which mice entered were recorded in order to calculate the percentage of spontaneous alteration. The number of alternations was counted when the mouse entered into the three different maze arms consecutively. The percent of spontaneous alteration was calculated as the number of alterations divided by the total entry number multiplied by 100 . 


\section{RT-PCR analysis}

To verify the amount of mouse or human tau mRNA levels in $\mathrm{ADLP}^{\mathrm{Tau}}$ and $\mathrm{ADLP}^{\mathrm{APT}}$ mice, RT-PCR analysis was carried out with 10-month-old ADLP model mice. Total RNA was extracted from the hippocampus with the RNeasy Mini kit (QIAGEN). All RNA samples were converted into cDNA using Maxime RT PreMix Kit (iNtRON BIOTECH). Quantitative RT-PCR was carried out in triplicates using KAPA SYBR FAST ABI Prism qPCR kit (KAPA Biosystems). For mouse Tau, the primers 5'AGCCCTAAGACTCCTCCA-3' and 5'-TGCTGTAGCC GCTTCGTTCT-3' were used. Human tau was amplified with the primers 5'-CTCCAAAATCAGGGGATCGC-3' and 5'-CCTTGCTCAGGTC AACTGGT-3'. The mRNA levels of mouse Tau and human Tau were normalized with GAPDH which was amplified with the primers 5'GGCCTTGACTGTGCCGTTGAATTT-3' and 5'-ACA GCCGCATC TTCTTGTGCAGTG-3'. Once the reaction was completed, the RT-PCR products were evaluated/analyzed via gel-electrophoresis to measure/ calculate the specificity of human tau primers.

\section{Western blot analysis}

Western blot analysis was used to confirm tau aggregates from sarkosyl-insoluble tau fractionation and the validation of proteomic analysis results. After isolated tau aggregates were heated at $70{ }^{\circ} \mathrm{C}$ for $10 \mathrm{~min}$ to prevent further tau protein aggregation, the same volume of sarkosyl-insoluble tau aggregate samples was loaded per lane of $4-12 \%$ Bis-Tris polyacrylamide precast gels (NuPAGE system, Invitrogen). Following electrophoresis, proteins were transferred to a PVDF membrane. Membranes were blocked with 5\% skim milk solution and then incubated with primary antibodies against human tau (Tau13, Abcam, 1:1000) and total tau (endogenous tau and human tau) (Tau5, Abcam, 1:1000). Primary antibodies against ABCA1 (Abcam, 1:500), Ptprc (CD45, Abcam, 1:1000) and Hcls1 (HS1, CST, 1:1000) were used for the validation of proteomic analysis. For confirmation of kinase expression levels, CK1 $\delta$ (Abcam, 1:5000), RSK1 (CST, 1:2000) and GSK-3 $\beta$ (CST, 1:2000) were used as primary antibodies. Anti-mouse or rabbit IgG conjugated HRP was used to detect primary antibodies and West Save Gold (Ab frontier) was used for their visualization. Since sarkosyl-insoluble fractionation only extracts protein aggregates, certain proteins generally used for normalization such as GAPDH and $\beta$-actin were not detected in the sarkosyl-insoluble pellets. Thus, total antibody signals of tau aggregates or each signal for distinct sizes of tau aggregates were quantified for quantification.

\section{Mass spectrometry-based proteomics}

Mouse hippocampus tissues were resected and subjected to the previously described sample preparation methods with some modifications [18-20]. Detailed procedures including protein digestion, peptide labeling, fractionation, and MS analysis are described in Additional file 1: Supplementary Methods.

\section{Quantification of protein abundance and statistics}

The quantification and statistical processing methods described below are related to proteomic data. First of all, among the 9814 identified proteins, only 6964 proteins satisfying the following criteria were used for subsequent quantitative analysis; identification in all channels (7022 proteins), high protein confidence (6970 proteins, assessed by Proteome Discoverer), and possessing 1 or more unique peptides (6964 proteins). The protein abundance ratio of individual samples to pooled sample (named "normalized protein abundance") was generated by dividing the reporter ion intensity of each channel by the intensity of the pooled sample channel in its corresponding experimental TMT set (Additional file 2: Figure S3B). There was no significant difference between the values of the pooled sample channels (Additional file 2: Figure S5E). Thus, the denominators were considered to be common and eliminated. The fold-change values used in the bioinformatics analysis were generated by dividing the normalized protein abundance of each transgenic mouse by the value of the age-matched wild type mouse. The distribution of ratiometric data was almost normal (Additional file 2: Figure S5G) but this was not thoroughly tested.

Statistical processes for the proteomic data were performed based on the normalized protein abundance using Perseus [21]. Initially, total identified proteins were filtered based on the 6964 proteins that quantified in all mouse samples. The statistical cut-off value for significance was set to $p$-value $<0.05$ for the Student's t-test, while Benjamini-Hochberg FDR adjusted p-value cut-off [22] of 0.05 was applied for the ANOVA test. The normalized protein abundances were subjected to znormalization followed by hierarchical clustering. The statistical tests for the other biochemical experiments were described in each figure legend.

\section{Bioinformatics analysis}

The Gene Ontology (GO) of the proteins was classified using DAVID bioinformatics tool (version 6.8) [23]. The GO classification was evaluated by Fisher's exact test to obtain a set of $P$-values, which were then filtered at a cutoff value of 0.01 . Canonical pathways, downstream biological functions, and upstream regulators were enriched using Ingenuity Pathway Analysis (IPA, QIAGEN) [24]. The analytical algorithms embedded in IPA uses input protein list (here differentially expressed proteins) to predict putative upstream regulators such as transcription factors and growth factors, as well as downstream effects on known biological pathways. IPA derives these protein 
set-pathway (or regulator) relationships from their own large-scale causal network database, named Ingenuity Knowledge Base. Because the algorithm cannot determine with certainty which causalities in its database can explain our experimental results, the tool performs statistical tests (i.e. Fisher's exact test) to assess the reliability of predicted upstream genes and pathways. Finally, IPA also assigns activation states (activated or inhibited) to putative regulators or pathways based on the quantitative values of protein members. The user will be given a confidence in the $P$-value obtained from the Fisher's exact test and the magnitude of activation as a Z-score, respectively. In this study, the $P$-value cut-off criteria for the enrichment was 0.01 for Fig. $6 \mathrm{a}$ and $\mathrm{b}$ and the predictive activation $\mathrm{Z}$-score cut-off was 1 . For the Fig. $4 \mathrm{~b}$, the cut-off value was 0.05 and the predictive activation Z-score cut-off was 1 . The initial pathway diagrams were obtained by IPA but were manually modified. Protein-protein interactions (PPIs) for the network analysis was interrogated from STRING database (http://www.string-db.org) [25]. The PPIs in network model were visualized using Cytoscape [26].

\section{Results}

\section{Pathological symptoms of ADLPAPT mice}

Four mouse models with the same genetic background were generated: wild-type, ADLP ${ }^{\mathrm{APP} / \mathrm{PS} 1}$ (A $\beta$ accumulation only), ADLP ${ }^{\text {Tau }}$ (NFTs only), and ADLP ${ }^{\text {APT }}$ (both $A \beta$ and NFTs) mice. To confirm the amyloid plaque burden in the hippocampus of the ADLP mice, we stained coronal brain sections of 4-, 7-, and 10-month-old ADLP mice with the biotin-4G8 antibody that recognizes amino acids $17-24$ of $\mathrm{A} \beta$ peptides. Both $A D L P^{A P P / P S 1}$ and $\mathrm{ADLP}^{\mathrm{APT}}$ mice started showing slight extracellular accumulation of $\mathrm{A} \beta$ at 4 months and exhibited large amyloid plaques at 7 months; no significance was observed between-model difference in their amyloid plaque burdens (Fig. 1a and c).

To investigate the NFT pathology in the hippocampus, we first stained phosphorylated tau using the AT8 antibody, which detects residue Ser202/ Thr205 of phosphorylated tau in paired helical filaments (PHFs). Intense immunoreactivity of PHFs against phosphorylated tau was observed in the hippocampal CA1 area of 7- and 10-month-old ADLP ${ }^{\text {APT }}$ mice compared with ADLP $^{\text {Tau }}$ mice (Fig. $1 \mathrm{~b}$ and $\mathrm{d})$. Subsequently, we used the AT180 antibody to detect the residue Thr231 of phosphorylated tau. The residue Thr231 was strongly stained at the soma and dendrites of CA1 neurons starting from 4 months of age. In 10-month-old mice, we observed phosphorylated tau localized only at the soma region as a form of NFTs, indicating that ADLP ${ }^{\mathrm{APT}}$ mice developed severe NFT pathology compared with $\mathrm{ADLP}^{\mathrm{Tau}}$ mice (Additional file 2: Figure S1A).
To validate the expression of tau in the hippocampus, mRNA levels of endogenous mouse tau were confirmed by RT-PCR analysis. The results indicated no significant differences among the ADLP mouse models. Furthermore, the human tau mRNA expression was not different between ADLP $^{\text {Tau }}$ and ADLP ${ }^{\mathrm{APT}}$ mice, indicating accelerated NFT pathology in ADLP ${ }^{\mathrm{APT}}$ mice without quantitative difference in tau protein (Additional file 2: Figure S1B-1D). Subsequently, we isolated NFTs from the hippocampus of 7- and 10-month-old ADLP ${ }^{\mathrm{Tau}}$ and ADLP ${ }^{\mathrm{APT}}$ mice using sarkosyl-insoluble tau fractionation. Tau aggregates in $\mathrm{ADLP}^{\mathrm{APT}}$ mice were composed of higher molecular weight of tau at both 7 and 10 months of age, and they appeared at regular intervals above the $55 \mathrm{kDa}$ of human tau. The proportion of the higher molecular weight tau increased in 10-month-old ADLP ${ }^{\mathrm{APT}}$ mice as compared to agematched ADLP ${ }^{\text {Tau }}$ mice (Fig. 1e). However, ADLP ${ }^{\text {Tau }}$ mice had only low-molecular-weight tau as a main constituent until 10 months of age (Fig. 1e and f). This suggests that the phosphorylation of human tau was increased by the amyloid pathology in ADLP ${ }^{A P T}$ mice. Using the Tau5 antibody, which reacts to both mouse and human tau, we confirmed that sarkosyl-insoluble tau fractionation was the appropriate method for specifically extracting tau aggregates from the hippocampus of ADLP ${ }^{\text {Tau }}$ and ADLP ${ }^{\mathrm{APT}}$ mice (Additional file 2: Figure S1E).

We also examined whether the accelerated NFT pathology observed in the hippocampal CA1 area of ADLP ${ }^{\mathrm{APT}}$ mice was associated with neuronal loss in the CA1 layer. Neuronal quantification using NeuN immunostaining revealed a significant decrease in the number of CA1 neurons in 10-month-old ADLP ${ }^{\mathrm{APT}}$ mice, but not in the other models, indicating that the amyloid pathology of this model aggravated neuronal death via the NFT pathology (Fig. $1 \mathrm{~g}$ and $\mathrm{h}$ ). Because spatial memory impairment is an essential feature in AD animal models [27], we used the alternation Y-maze task to evaluate spatial memory in ADLP mice at different ages. Although there was no memory deficit in 4-month-old ADLP ${ }^{A P T}$ mice, they showed significant deficits in spatial memory beginning at 7 months (Fig. 1i). Altogether, these data indicated that ADLP $^{\text {APT }}$ mice exhibit an accelerated NFT pathology, amyloid pathology, neuronal loss, and age-related memory impairment. Thus, this model appears to comprehensively reflect the pathophysiological changes in AD.

\section{Constructing a Hippocampal proteome database of the ADLP mouse models}

To obtain insights into the molecular basis of AD pathogenesis, we performed quantitative proteomic analysis using three replicates of hippocampus resected from each mouse model at three time points $(4,7$, and 10 months of age; Fig. 2a). Briefly, each hippocampus was homogenized and digested via filter-associated sample preparation [28], 


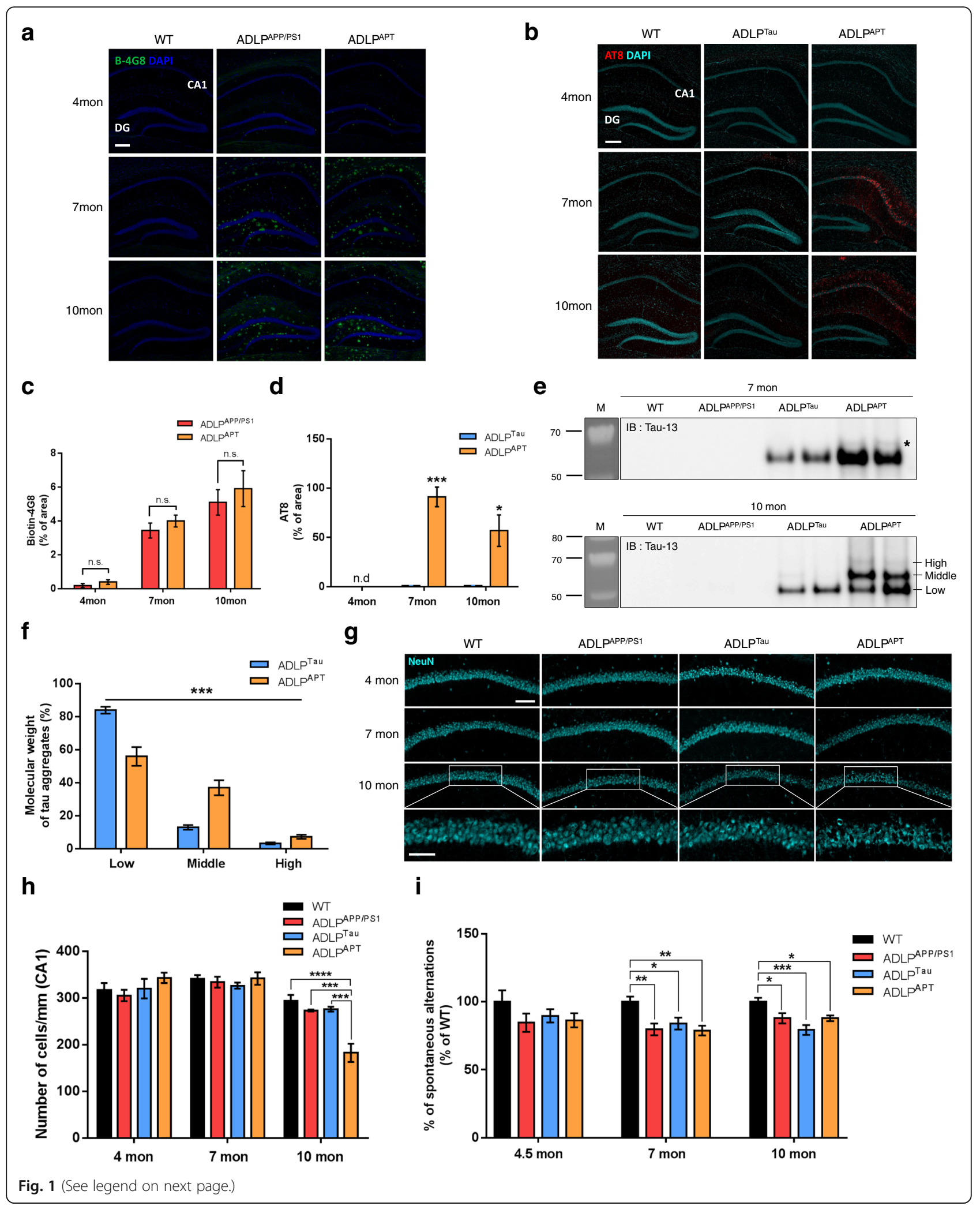


(See figure on previous page.)

Fig. 1 Pathological characterization of a novel animal model of Alzheimer's disease. a and c For detection of amyloid plaques, hippocampal regions of ADLP mice were immunostained with the biotin-4G8 antibody at 4-, 7-, and 10-month-old. The percentage of amyloid plaques area was not significantly different between ADLP ${ }^{A P P / P S 1}$ and ADLPAPT mice (Student's t-test, $n=3-4$ per group). Scale bar represents $200 \mu \mathrm{m}$. $\mathbf{b}$ and $\mathbf{d}$ ) The hippocampal CA1 layer of ADLP mice was stained with the AT8 antibody against phosphorylated tau (Ser202/Thr205). A significant increase in AT8 immunoreactivity was observed in ADLP APT mice compared with age-matched ADLP ${ }^{\text {Tau }}$ mice (Student's t-test, $n=3-4$ per group). Scale bar represents $200 \mu \mathrm{m}$. e Sarkosyl-insoluble tau fractions from 7 and 10 months ADLP mice hippocampus were analyzed by western blot analysis using human tau specific antibody (Tau13). f Each distinct size of sarkosyl-insoluble tau was quantified in 10 months old ADLP Tau and ADLP ${ }^{\text {APT }}$ mice (Chisquare test; $n=6$ mice per genotypes). $\mathbf{g}$ The CA1 pyramidal neurons of ADLP mice were stained with anti-NeuN antibody to determine degrees of neuronal loss. Scale bars represents $100 \mu \mathrm{m}$ or $50 \mu \mathrm{m}$ (enlarged figures). $\mathbf{h}$ Quantification of the number of CA1 neurons in 7-and 10-month-old ADLP mouse model (one-way ANOVA in each age of ADLP mouse model). i ADLP model mice showed memory impairment compared with wild-type mice, which examined by the Y-maze test (one-way ANOVA in each age of ADLP mouse model, $n=9-11$ per group). Results are expressed as mean \pm SEM. ${ }^{*} P<0.05$, ${ }^{* *} P<0.01,{ }^{* * *} P<0.001,{ }^{* * * *} P<0.0001$

and the peptide samples were labeled with TMT reagents. The labeled samples were analyzed using Q Exactive mass spectrometer. The resulting 96 raw files (12 peptide fractions $\times 4$ TMT-mix experimental sets $\times$ technical duplicates) were processed in Proteome Discoverer based on the SEQUEST-HT algorithm. In total, 9814 protein groups were identified from 125,683 unique peptides; among them, 7022 protein groups were successfully identified in all 36 samples (Fig. 2b and c). The expression levels of the mutated human proteins (APP, PSEN1, and tau) were clearly verified by our MS analysis (Additional file 2: Figure S1F-H). Our proteomic platform allowed us to discover in-depth proteome which dynamic range covered over six orders of magnitude. This comprehensive dataset included a number of well-known risk factors of $\mathrm{AD}$ (http://www.alzgene.org), such as Apoe [29], Clu [30], and TDP-43 (Tardbp) [31,32], which were quantified in all samples. Notably, we also detected low-abundance risk factors, such as Gab2 [33-35], Trem2 [36], and Abca7 [37], indicating that our proteome exhibited a profound quantification depth (Fig. 2d). The other genes shown in Fig. 2d were reported as AD risk factors in other studies [38-47]. Thus, our extensive proteome profiling of the mouse hippocampus yielded a database that could form a solid foundation for further biological interpretations. All protein identification data are presented in Additional file 3: Table S1. The proteins quantified in all 36 channels are listed in Additional file 4: Table S2. In addition, we have provided the interactive plot that shows the protein changes over three age points for the input gene in "Profile plot" sheet of Additional file 4: Table S2 [48].

To assess the comprehensiveness of the proteome data, we compared our data with those in a recently reported mouse brain proteome atlas (http://www.mousebrainproteome.com) [9]. The comparative analysis showed that approximately $87 \%$ of the protein groups in this dataset, which is the largest to date, were also identified in our study. Notably, despite using less instrument time than the label-free method used in constructing the mouse brain atlas, our TMT-based proteomics technique has constructed a proteome dataset of comparable depth. Furthermore, 2344 genes were uniquely identified in our dataset, indicating that our data expanded the hippocampal proteome to a great extent using a single type of sample on a more time-efficient platform (Additional file 2: Figure S4A). In addition, we compared the efficiency of protein identification with those reported in other proteomic studies of transgenic AD mouse models; our dataset contained the highest number of protein identifications (Additional file 2: Figure S4B).

\section{Quality assessment of the proteomic data}

The multiplexing capacities of the TMT-based workflow allowed us to characterize the quantitative variations within and between hippocampal samples. Our analysis of a non-homologous (i.e., ovalbumin) spiked-in standard for inter- and intrabatch normalization revealed a coefficient of variation of $6.3 \%$ during repetitive MS analysis (Additional file 2: Figure S5A). Although the intensity variations among non-normalized biological replicates showed a suitable reproducibility, we observed a slight improvement of reproducibility when the normalization was performed using ovalbumin (Additional file 2: Figure S5B and C). Thus, the reporter ion intensities of proteins were initially normalized with respect to the intensity of ovalbumin.

To investigate the quantitative reproducibility (technical and experimental set-to-set variations), we used protein abundances in pooled sample channels (131) to perform cross-correlation examinations. Based on the results, the duplicate MS analysis showed excellent consistency, with an average $R^{2}$ correlation value of 0.994 (Additional file 2: Figure S5D). The mean $R^{2}$ value of the quantified protein abundance between the TMT experimental sets was excellent at 0.992 (range, 0.988-0.995) (Additional file 2: Figure S5E). Furthermore, the results indicated good correlation among the biological replicates, with a mean Pearson's correlation value of 0.991 (Additional file 2: Figure S5F). Thus, the observed differences in protein expression reflected the molecular diversity among the mouse histotypes, not the effect of our TMT-based strategy [49]. 
a

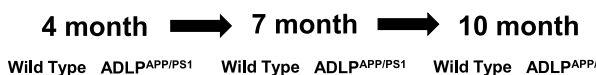
Wild Type ADLP ADLPTau ADLPAPT ADLPTau ADLPAPT ADLPTau ADLPAPT
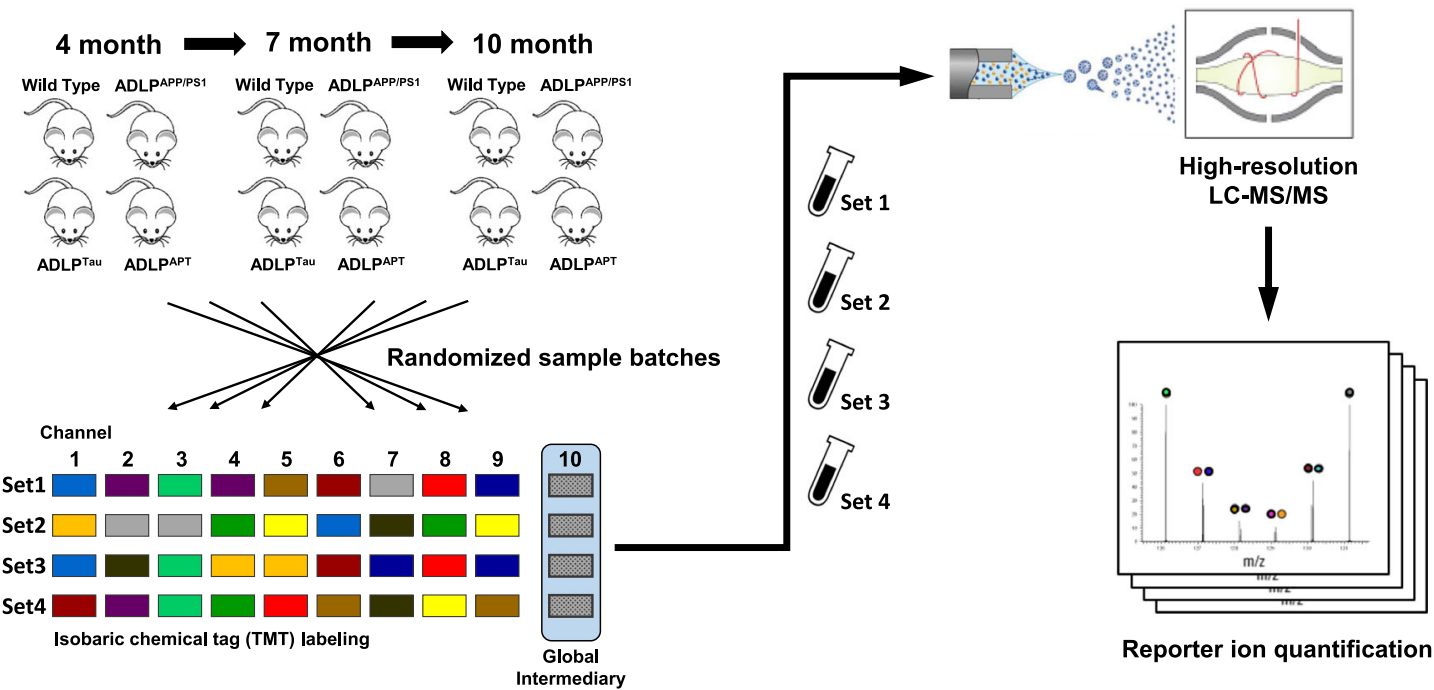

Reporter ion quantification

b

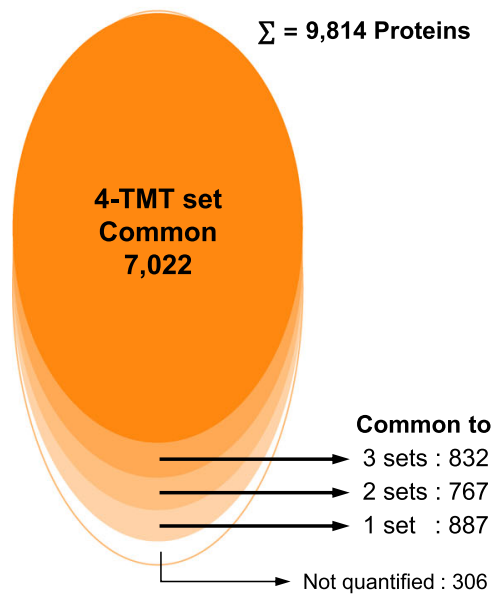

d

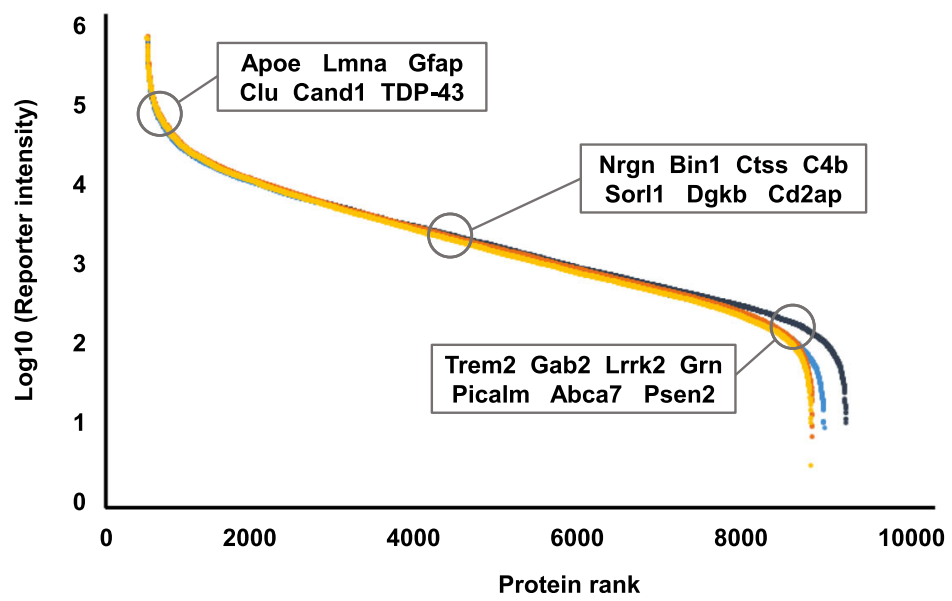

C

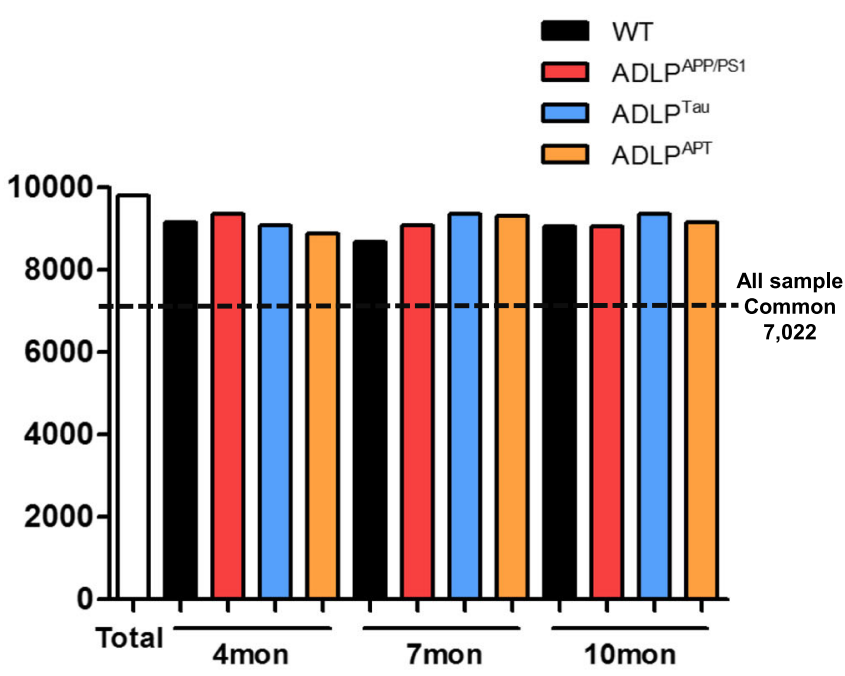

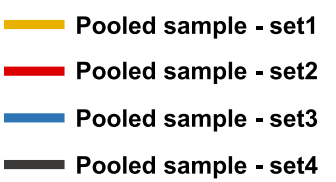

Fig. 2 (See legend on next page.) 
(See figure on previous page.)

Fig. 2 Hippocampal proteome analysis of ADLP model mice. a Graphical illustration of the workflow used for our TMT-based proteomic analysis. The detailed sample assignments for TMT labeling are shown in Fig. S2A. b A total of 9814 protein groups were identified in our study; of them, 7022 protein groups were identified and quantified in all experimental sets. The "Not quantified" proteins were identified via search algorithm, but their reporter ions were not detected and were excluded from subsequent quantitative analysis. c A bar chart showing the number of proteins identified in the hippocampus of each ADLP model. $\mathbf{d}$ Dynamic range of protein abundance, spanning six orders of magnitude. Normalized reporter ion intensities of pooled samples in each experimental set were used. Proteins known as AD risk factors are annotated in the plot

\section{Proteome alterations in ADLP hippocampus}

To resolve the regulated proteins in each ADLP mouse model, we quantitatively analyzed the protein expression in a ratiometric manner (see Methods). Based on the foldchange in the normalized protein abundance, we identified 63,178 , and 245 proteins that were significantly regulated in 4-, 7-, and 10-month-old ADLP ${ }^{\mathrm{APP} / \mathrm{PS} 1}$ mice, respectively (Student's $t$-test, $p$-value $<0.05$, fold-change $>1.25$ ). In comparison, 3, 42, and 4 proteins were significantly regulated in 4-, 7-, and 10-month-old ADLP ${ }^{\text {Tau }}$ mice, respectively, whereas 62, 131, and 311 proteins were significantly regulated in 4-, 7-, and 10-month-old ADLP ${ }^{\mathrm{APT}}$ mice, respectively. The scatter plots showed that more proteins were up-regulated during the progression of amyloid pathology than during that of NFT pathology. Interestingly, the overall tendencies of the significantly regulated proteins were similar in $\mathrm{ADLP}^{\mathrm{APP} / \mathrm{PS} 1}$ and $\mathrm{ADLP}^{\mathrm{APT}}$ mice, indicating that the amyloid pathology exerted stronger effects on the overall proteome than did the NFT pathology (Fig. 3). The proteins with significant change in each model are summarized in Additional file 5: Table S3.

To identify the specific protein changes under the various AD pathologies ( $A \beta$, Tau, or both $A \beta$ and Tau), we used ANOVA to investigate the global diversity. Our ANOVA indicated that 1094 protein groups were differentially expressed proteins (DEPs) among the ADLP mouse models (FDR cut-off <0.05; Additional file 6: Table S4). Hierarchical clustering divided these proteins into three groups, including up-regulated proteins (cluster 2; 681 proteins) and down-regulated proteins (cluster 3; 121 proteins). ADLP ${ }^{\text {Tau }}$ mice had relatively few DEPs, whereas ADLP APP/PS1 $^{\text {and }}$ $\mathrm{ADLP}^{\mathrm{APT}}$ mice shared DEPs that showed drastic agerelated perturbations (Fig. 4a). This suggests that tau itself is insufficient to drive the hippocampal proteome regulation during the stage up to 10 months of $\mathrm{AD}$ pathogenesis.

We subsequently investigated how these DEPs affected downstream pathways in an unbiased manner. The ingenuity pathway analysis (IPA) platform identified five canonical pathway categories (nervous system signaling, immune response, cell cycle/apoptosis, cancer/disease signaling, and intracellular signaling pathways) that were significantly enriched by the DEPs ( $p$-value $<0.05$; activation Z-score $>1$; Fig. 4b). The primary enriched pathway categories were nervous system signaling and the immune response, which are known to be associated with $\mathrm{AD}$ pathogenesis. While the signaling pathways of the nervous system tend to become inactive with age, most identified immune response pathways were predicted to be activated as the disease progressed. The exact Zscores and $p$-values for the IPA results are listed in Additional file 7: Table S5.

\section{Identifying molecular and functional signatures associated with pathology of ADLP ${ }^{\text {APT }}$ mice}

We next investigated the age-dependent dysregulation of biological functions in ADLP ${ }^{\mathrm{APT}}$ mice. We identified 732 proteins that were differentially expressed with respect to ADLP ${ }^{\mathrm{WT}}$ mice (DEPs ${ }^{\mathrm{APT}}$, ANOVA FDR cut-off <0.05; Additional file 8: Table S6). Our hierarchical clustering analysis divided the DEPs ${ }^{\mathrm{APT}}$ into five clusters, including gradually down-regulated proteins (cluster 2; 79 proteins) and gradually up-regulated proteins (cluster 5; 499 proteins; Fig. 5a). Gene ontology (GO) enrichment analysis revealed that the proteins in cluster 2 were particularly involved in synapses and cytoskeleton binding, suggesting that the AD pathologies gradually damage the synaptic plasticity in the hippocampus of ADLP ${ }^{\mathrm{APT}}$ mice (Fig. 5a, right panel, and Fig 5b). The proteins of cluster 5 were primarily assigned to the inflammatory and degradation systems, such as leukocyte-mediated immunity, phagocytosis, endosomes, and lysosomes, indicating that AD pathologies could activate both inflammation and degradation systems to remove $A \beta$ plaques and NFTs (Fig. 5c and Additional file 9: Table S7).

To define biological activities that contribute to the pathological phenotypes of ADLPAPT mice, we performed bioinformatics analysis using IPA to identify upstream regulators as well as downstream functions of $\mathrm{DEPs}^{\mathrm{APT}}$ (Additional file 10: Table S8). The IPA predicted 25 relevant upstream regulators to modulate $\mathrm{DEPs}^{\mathrm{APT}}$. In addition, DEPs ${ }^{\mathrm{APT}}$ were assigned to 27 downstream biological functions associated with the immune response and the nervous system (p-value $<0.01$; Fig. 6a). To further explore the predictive biological pathways significantly altered in ADLP ${ }^{\mathrm{APT}}$ mice, we investigated relevant canonical pathways ( $p$-value $<0.01$; Additional file 10: Table S8). Among these, one of the most dysregulated immune response-related pathways was the leukocyte extravasation signaling pathway. Adhesion-related proteins, which are mainly expressed in peripheral immune cells and endothelial cells, were differentially expressed in 10-month-old ADLP ${ }^{\mathrm{APT}}$ mice 


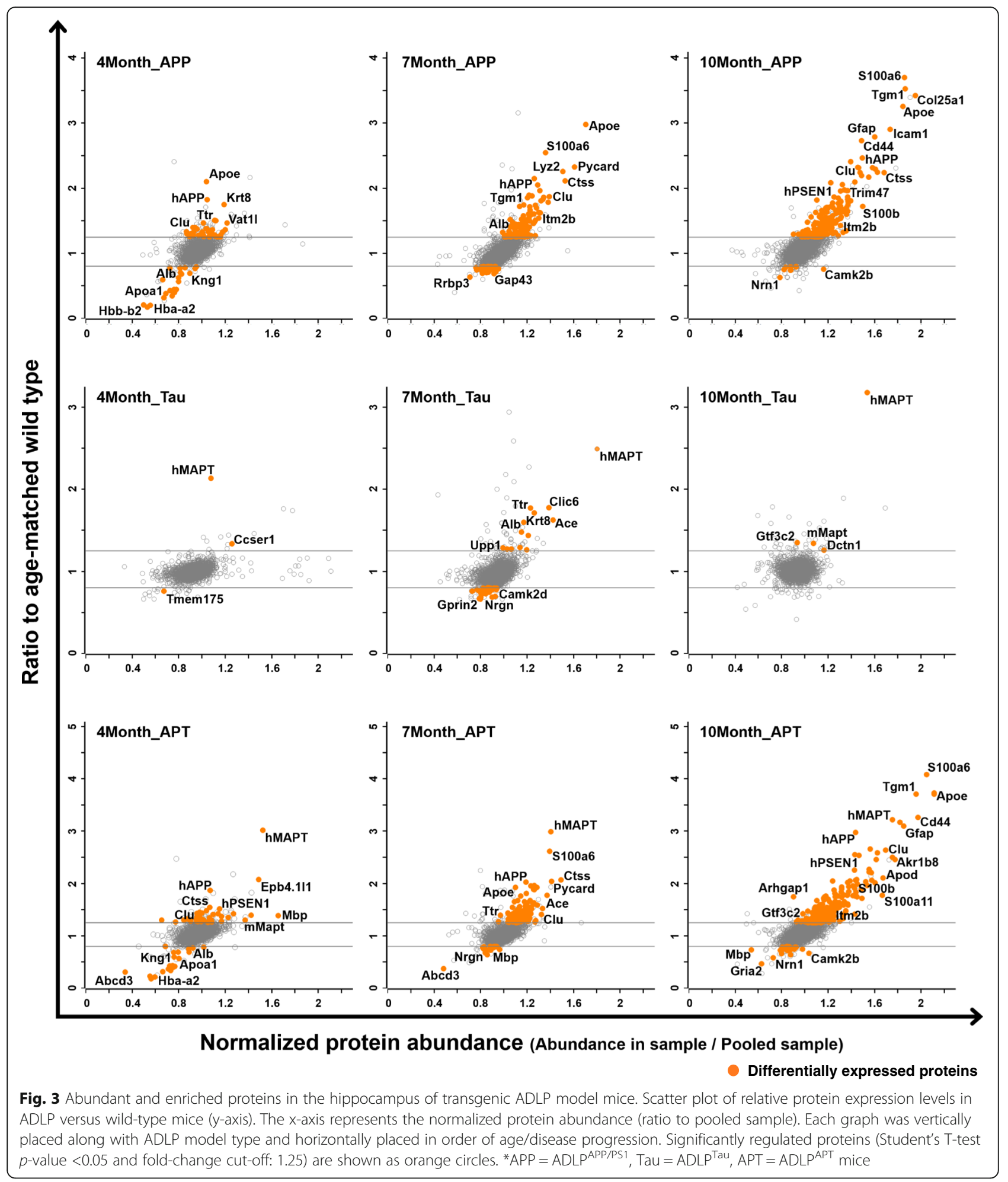

(Fig. 6b). We speculate that changes in endothelial cells are likely to reflect the $\mathrm{AD}$ pathology in the brain parenchyma and that such changes recruit peripheral immune cells into the brain parenchyma. Among the canonical pathways related to the nervous system, synaptic long- term potentiation (LTP) was a representative pathway altered in ADLP ${ }^{A P T}$ mice (Fig. 6c). Post-synaptic proteins of this pathway were mainly dysregulated by the extracellular amyloid pathology and the intracellular NFT pathology. Thus, the biological pathway alterations seen 


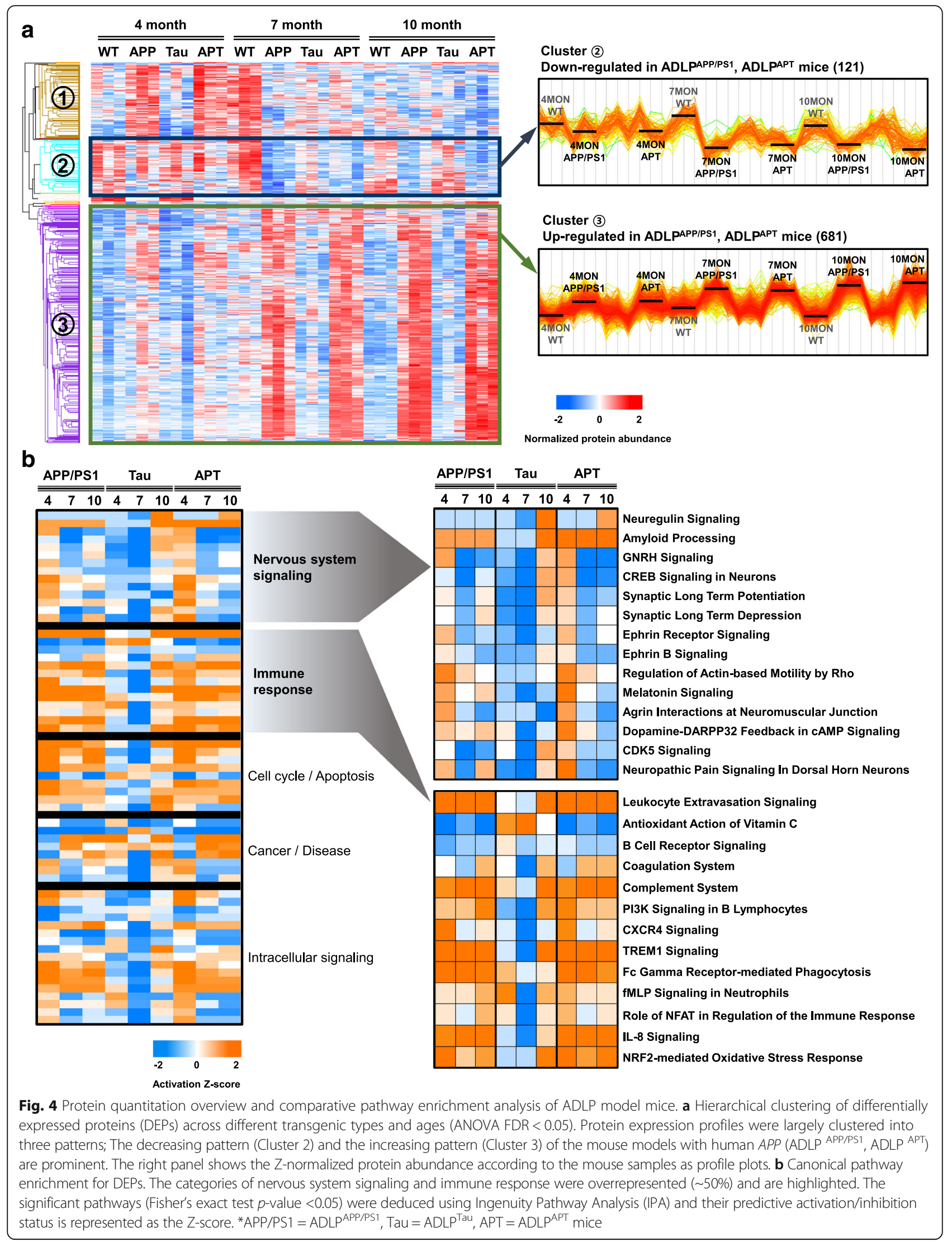




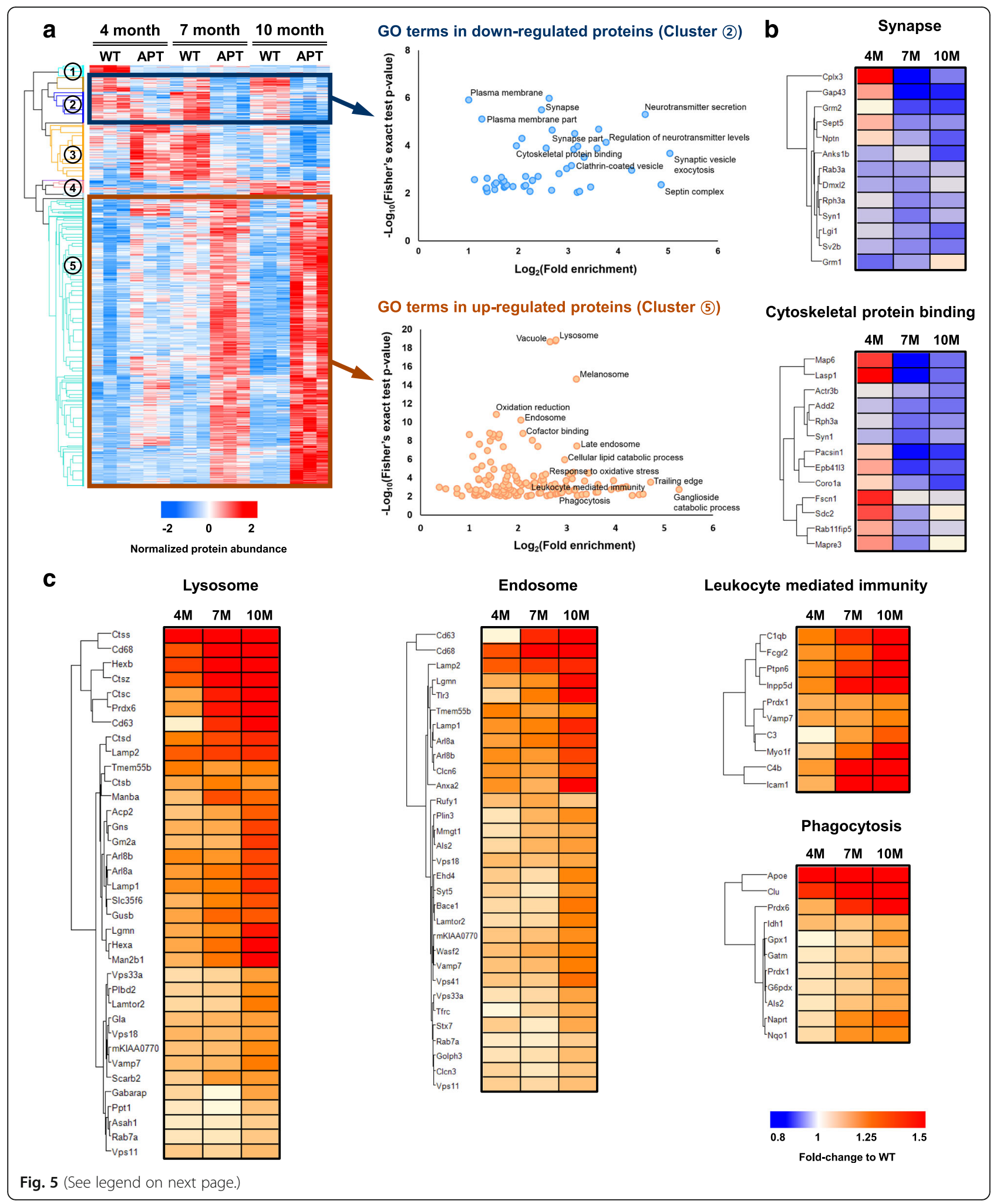


(See figure on previous page.)

Fig. 5 Protein clustering reveals major biological and molecular signatures of ADLPAPT mice. a Hierarchical clustering of differentially expressed proteins ( $\left(\mathrm{EP}^{\mathrm{APT}} \mathrm{s}\right.$ ) between ADLP APT and wild-type mice $(732$ proteins; ANOVA FDR $<0.05)$. The DEPS ${ }^{A P T}$ were clustered into five types of expression pattern. The protein expression level is the normalized protein abundance represented after Z-normalization. Scatter plot of enriched Gene Ontology (GO) terms in protein cluster 2 (down-regulated proteins) and cluster 5 (up-regulated proteins) are shown in the right panel. The -log 10 ( $P$-value) is plotted against the fold-enrichment of each $\mathrm{GO}$ term. $\mathbf{b}$ Heatmaps showing the expression levels of the proteins involved in the $\mathrm{GO}$ terms, such as synapse proteins and cytoskeleton binding proteins, in cluster 2 (down-regulated proteins). c Heatmaps for the expression levels of co-regulated proteins corresponding to the GO terms, such as lysosome, endosome, leukocyte-mediated immunity, and phagocytosis, in cluster 5 (up-regulated proteins)

in $\mathrm{ADLP}^{\mathrm{APT}}$ mice are consistent with the pathological changes seen in the nervous system of patients with AD.

\section{Network analysis of unique proteome changes in ADLP ${ }^{A P T}$ mice}

To identify ADLP ${ }^{A P T}$ mouse-specific protein changes, we investigated the exclusive DEPs ${ }^{\mathrm{APT}}$ in 4- (early) and 10-month-old (late) ADLP ${ }^{\mathrm{APT}}$ mice (Fig. $7 \mathrm{a}$ and Additional file 11: Table S9). Using the STRING database, we organized a protein-protein interaction map of the exclusive DEPs ${ }^{\mathrm{APT}}$ representing expression changes by age and the GO categories into which these proteins could be classified. Our GO analysis revealed that the exclusive $\mathrm{DEPs}^{\mathrm{APT}}$ were classified in the categories related to the immune response (e.g., cell-cell adhesion and inflammatory responses) and neuronal functions (e.g., syntaxin binding, lysosomes, and neuron projection development) (Fig. 7b). Subsequently, we examined the correlation of exclusive $\mathrm{DEPs}^{\mathrm{APT}}$ with 2 AD-causative molecules, App and Mapt, in an effort to depict how the amyloid and NFT pathologies interconnect with one another. The constructed App-Mapt network showed the direct or indirect connections of proteins with App and Mapt, suggesting that these molecular targets could influence specific pathologies of ADLP ${ }^{\mathrm{APT}}$ mice (Fig. 7c). The expression of Abca1, Ptprc, and Hcls1 that form a protein-protein interaction bridge between $A p p$ and Mapt were further validated by the western blot analysis (Fig. 7d). The expression levels of exclusive DEP$\mathrm{s}^{\mathrm{APT}}$ in the other transgenic mice are presented in Additional file 2: Figure S6.

\section{Comparison of ADLP ${ }^{A P T}$ mouse proteome with human AD proteome}

We ultimately aimed to construct a mouse model that fully recapitulates the molecular and functional features of $\mathrm{AD}$. To investigate how the $\mathrm{ADLP}^{\mathrm{APT}}$ mouse model reflects $\mathrm{AD}$, we performed a comparative analysis with the proteome data of a recently published human AD study [50]. This group performed proteomic analysis using brain tissues from patients with AD, elucidating molecular features and protein networks. Our dataset encompassed approximately $92 \%$ of the proteins identified in the human AD proteomics study. The expression patterns of DEPs common to both studies (Student's $t$-test $p$-value $<0.05$ ) overlapped by 41 and $25 \%$ in up-regulated and down- regulated proteins, respectively (Additional file 2: Figure $\mathrm{S} 4 \mathrm{C})$. Although the numerical correlation between the two data was low $\left(R^{2}<0.3\right)$, the commonly significant proteins have been classified into functional categories related to AD. Particularly, 10 up-regulated proteins, such as $\mathrm{Clu}$, Mao-b, Cyp46a1, and Vps26a, were deduced to have significant associations with AD (Additional file 12: Table S10). These results implicated that our mouse model adequately reflects the known molecular characteristics of $\mathrm{AD}$ and can thus be further utilized in the researches to discover new drug targets. Furthermore, the other proteins in the categories related to neurodegeneration may play a significant role in future studies. The results of the comparative analysis with human proteomic data are listed in Additional file 4: Table S2.

\section{Discussion}

The amyloid cascade hypothesis claims that $A \beta$ is the initial trigger for further pathological changes, including tau hyper-phosphorylation and NFT formation, which accelerate AD progression [51]. To investigate the prominent role of $A \beta$ in causing $A D$ pathogenesis, we developed ADLP ${ }^{\mathrm{APT}}$ mice, a novel animal model of AD that develops more robust amyloid and NFT pathologies than do other available animal models. This model showed no aggravation of the amyloid pathology beyond that seen in ADLP ${ }^{\mathrm{APP} / \mathrm{PS} 1}$ mice, indicating that the development of the NFT pathology does not affect the amyloid pathology. However, based on sarkosyl-insoluble tau fractionation for the aggregated form of tau and immunostaining for phosphorylated tau, the accelerated NFT pathology appeared in the hippocampus of ADLP ${ }^{\text {APT }}$ mice starting from 7 months of age in the absence of any observable difference in the transcription/expression of the human MAPT gene. Abnormal tau phosphorylation is a crucial event that triggers tau aggregation in AD brains [52]. Various kinases have been suggested to be involved in tau phosphorylation process. Among external stimuli to activate kinases, the role of $A \beta$ for activating tau kinases has been reported in a downstream of $A \beta$ toxicity [53]. In order to investigate whether increased kinase expression levels are causative factors for accelerating NFT pathology, we have confirmed the expression levels of three kinases (Csnk1d, Gsk3-beta and Rps6ka1) involved in tau phosphorylation by western 


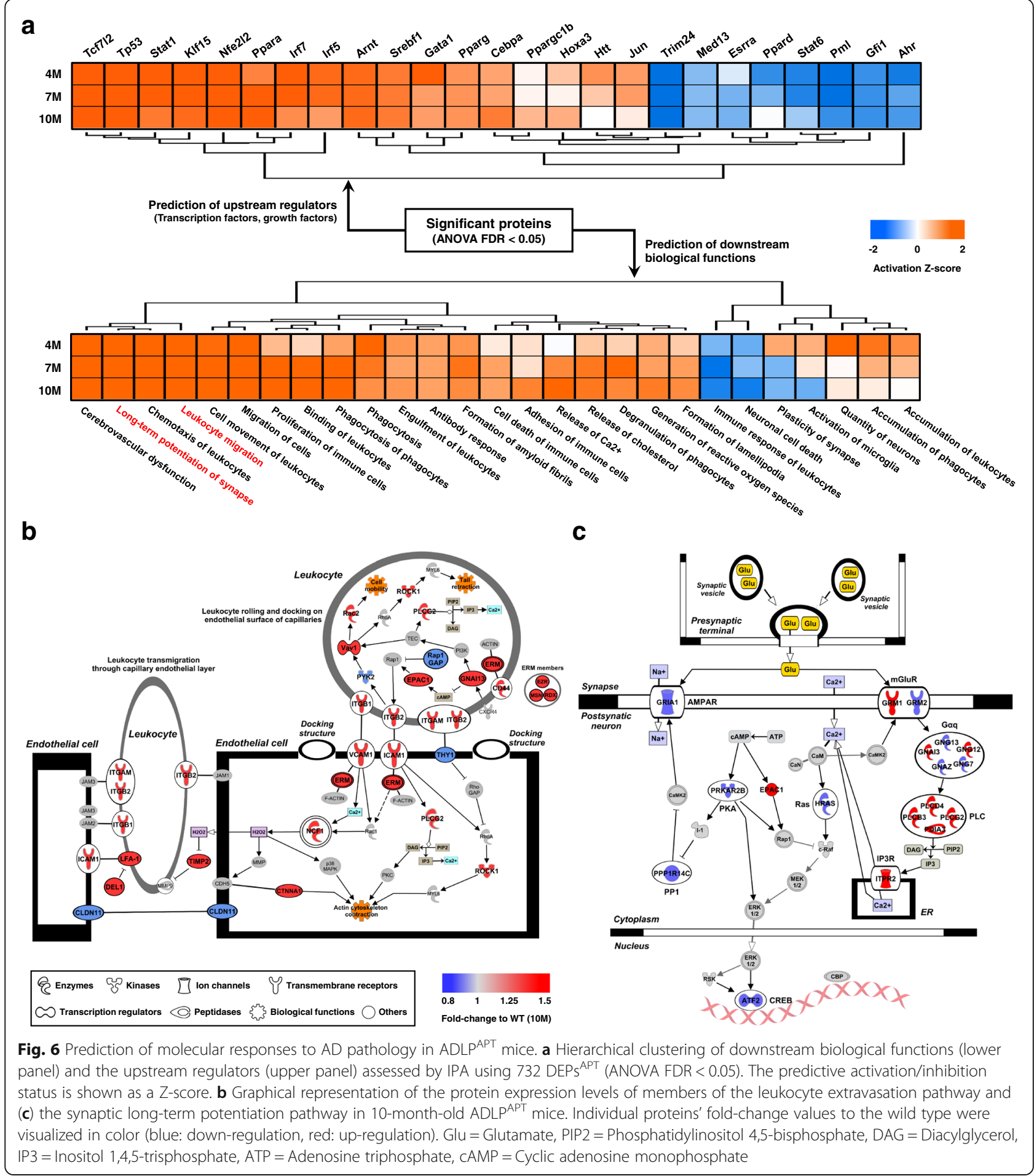

blot analysis [54-56]. Notably, no differences were observed in the kinase levels between wild-type and $\mathrm{ADLP}^{\mathrm{APT}}$ mice with the disease progression. In addition to western blot analysis, the MS results also indicated no changes in kinase expression levels. As for the kinases not checked by western blot analysis (e.g. Cdk5, Src, Fyn and Abl1), they were not changed among all groups, as confirmed by our hippocampal proteome data. These results support that accelerated NFT pathology in $\mathrm{ADLP}^{\mathrm{APT}}$ mice hippocampus is not accompanied by changes of tau kinases (Additional file 2: Figure S7). Previous studies have hypothesized that $A \beta$ is the initiator of the NFT pathology $[53,57,58]$. Indeed, $A \beta$ affects tau kinase activity or localization resulting in tau toxicity in 


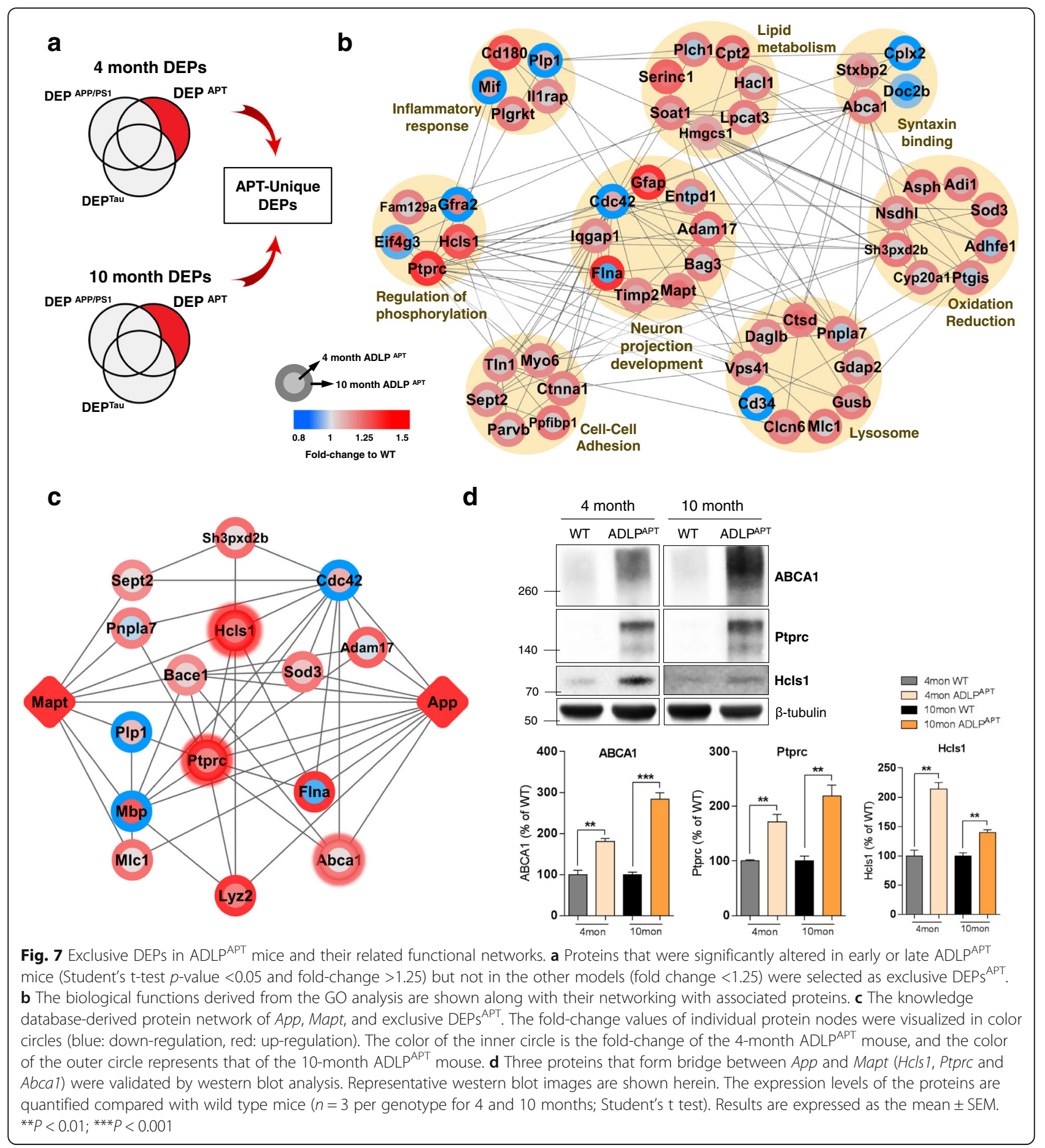

neurons. Moreover, the amyloid pathology in the cortex accelerates tau propagation process throughout the entorhinal cortex and aggravates the NFT pathology [57]. Because the underlying molecular mechanisms of the pathophysiological interaction between $A \beta$ and tau are unclear, ADLP ${ }^{\mathrm{APT}}$ mice may enable further exploration of the $A \beta$-tau axis hypothesis on the progression of $A D$ pathogenesis.
To further investigate the biological functions altered in the hippocampus of $\mathrm{ADLP}^{\mathrm{APT}}$ mice, we first constructed a hippocampal proteomic database of ADLP animal models using LC-MS. Notably, as the disease progressed, the protein expression profiles of ADLP $^{\text {Tau }}$ mice resembled those of wild type mice, indicating that the relatively slow NFT pathology had a small impact on the proteomic changes during the early stage of the 
disease. In contrast, ADLP $\mathrm{APT}^{\mathrm{T}}$ mice showed dramatic proteome-wide changes with a pattern similar to that of ADLP ${ }^{\mathrm{APP} / \mathrm{PS} 1}$ mice. One of the main explanations for the high similarity between ADLP ${ }^{\mathrm{APP} / \mathrm{PS} 1}$ and ADLP ${ }^{\mathrm{APT}}$ mice is the presence of inflammatory responses in the hippocampus. Almost all immune-related pathways exhibited similar changes over time in ADLP ${ }^{\mathrm{APP} / \mathrm{PS} 1}$ and ADLP ${ }^{\mathrm{APT}}$ mice, indicating that $A \beta$ is one of the main factors for active inflammatory responses in the hippocampus (Fig. 4b). In both mice, the immune response categories exhibited changes at 4 months of age, even before the first deposition of amyloid plaques in the hippocampus. Immunohistochemical analysis confirmed similarities in the inflammation status between ADLPAPP/PS1 and ADLP ${ }^{\mathrm{APT}}$ mice, as evidenced by the staining with astrocytes and microglia markers in the hippocampus (Additional file 2: Figure S2). These findings indicate that the immune responses in the hippocampus were mainly influenced by the amyloid pathology regardless of the NFT pathology.

Moreover, GO analysis of the gradually up-regulated DEPs in ADLP ${ }^{A P T}$ mice identified many glial cell typerelated categories, such as phagocytosis, cell chemotaxis, and macrophage infiltration (cluster 5, Fig. 5a). One of the main dysregulated pathways in ADLP ${ }^{\mathrm{APT}}$ mice was the leukocyte extravasation signaling pathway. Various adhesion-related molecules (Icam-1, Vcam-1, Itgb-1/2, and Cd44) were up-regulated in the hippocampus of ADLP ${ }^{\mathrm{APT}}$ mice (Fig. 6a and b), indicating that $\mathrm{AD}$ pathologies can modulate endothelial cells in the brain parenchyma and recruit peripheral immune [59]. Both amyloid plaques and neurofibrillary tangles can activate inflammatory responses, thereby inducing the secretion of cytokines from microglia and astrocytes. Moreover, these serial pathologies also affect endothelial cells and disrupt the blood-brain barrier (BBB) integrity in $\mathrm{AD}$ [60]. A disrupted $\mathrm{BBB}$ with increased inflammatory cytokines finally results in the recruitment of peripheral immune cells toward brain parenchyma in AD. Several previous reports have suggested communication between peripheral immune cells and brain parenchyma as a pathological phenotype of $\mathrm{AD}$, even if their functional roles remain unclear. Laurent $\mathrm{C}$ et al. have reported that CD8positive $\mathrm{T}$ cells infiltrate the hippocampus. They have also demonstrated that the infiltration of CD8-positive lymphocytes in the cortical region of patients with frontotemporal dementia who develop tauopathy resulted from P301L mutation within the tau gene [61]. Previously, using twophoton microscopy, we have observed that neutrophils can extravasate from blood vessels into the brain parenchyma where amyloid plaques are present. In the brain parenchyma, the neutrophils adhere to amyloid plaques and phagocyte them [62]. Moreover, Zenaro E et al. have reported that the number of neutrophils adhere both inside blood vessels and in the brain parenchyma of patients with AD [63]. Depleting temporarily neutrophils at the early stage of disease progression in a transgenic mouse model of $\mathrm{AD}$ prevented memory impairments, suggesting that the infiltration of neutrophils contributes to the pathogenesis of $A D$. The up-regulation of the proteins involved in the leukocyte extravasation signaling in ADLP ${ }^{\mathrm{APT}}$ mice suggested that peripheral immune cells may interact with capillary endothelial cells for infiltration into the brain parenchyma.

When glial or peripheral immune cells detect extracellular amyloid plaques, they clear the amyloid deposits through phagocytosis [64]. In addition to extracellular amyloid plaques, when neurons host NFTs in the cytosol, they activate the degradation system including the autophagy-lysosome system to eliminate them $[65,66]$. Consistent with these, the GO analysis revealed that the gradually upregulated DEPs ${ }^{\mathrm{APT}}$ included many components of the degradation system (cluster 5, Fig. 5c). In addition, constituents of the endosome GO category were upregulated, demonstrating that the endocytic pathway via endocytosis was also activated in ADLP ${ }^{\mathrm{APT}}$ mice. Thus, although we could not clarify which cell types exhibited these protein changes, the results of the GO analysis of upregulated proteins collectively suggested that $\mathrm{AD}$ pathologies trigger a sequential response that moves from phagocytosis to degradation via the endocytic pathway.

Several nervous system signaling pathways appear to differ between ADLPAPP/PS1 and ADLP ${ }^{\mathrm{APT}}$ mice. The two canonical pathways, cAMP response element-binding protein (CREB) signaling in neurons and synaptic LTP, were inactivated only in the 10 -month-old ADLP ${ }^{\mathrm{APT}}$ mice (Fig. 6c). It is established that CREB signaling contributes to cognitive functions by modulating synaptic plasticity [67]. Proteins associated with LTP processes are increased by CREB signaling in the hippocampus; in $\mathrm{AD}$, alterations in $\mathrm{Ca}^{2+}$ signaling lead to decreased CREB signaling and altered LTP [68]. Similarly, our ADLP ${ }^{\mathrm{APT}}$ mice showed downregulated CREB signaling and LTP compared with ADLP ${ }^{\text {Tau }}$ and $\mathrm{ADLP}^{\mathrm{APP} / \mathrm{PS} 1}$ mice. Thus, these two pathways are predicted to be disturbed simultaneously by both $A \beta$ accumulation and NFTs. This suggests that ADLP ${ }^{\mathrm{APT}}$ mice have pathological symptoms similar to those of patients with AD [69].

To investigate the putative mechanisms underlying the molecular pathogenesis of ADLP ${ }^{\mathrm{APT}}$ mice, we sorted exclusive DEPs in ADLP ${ }^{A P T}$ to identify unique molecular alterations. The protein-protein interaction map of exclusive DEPs ${ }^{\mathrm{APT}}$ showed their direct or indirect interactions with each other and categories that each protein belongs to. We further investigated the molecular interactions of exclusive DEPs ${ }^{\mathrm{APT}}$ with $A p p$ and Mapt (Fig. 7c). The generated App-Mapt network contained 15 components that have been reported to interact among each other, although little is known about their relevance to the $\mathrm{A} \beta$-tau axis. For example, Ptprc is a well-known microglia marker that has a strong 
association with $A \beta$ oligomerization [70]. However, to our knowledge, the regulation of this protein in the hippocampus of AD mouse models is not well studied. In contrast, $A b c a 1$, a risk factor for $\mathrm{AD}$, has been reported to play a significant role in $A \beta$ clearance [71]. In addition, Hcls1 is a leukocyte-specific actin-binding protein involved in immune response mechanisms [72]. We found that these proteins are involved in immune responses in the hippocampus, forming an interaction bridge on our App-Mapt network. Furthermore, western blot analysis clearly confirmed the regulation of these proteins in $\mathrm{ADLP}^{\mathrm{APT}}$ mice. Since our App-Mapt protein network provides information on DEPs affected by both $\mathrm{A} \beta$ and tau, it may help researchers to identify novel molecular mechanisms in the $\mathrm{A} \beta$-tau axis.

To verify whether $\mathrm{ADLP}^{\mathrm{APT}}$ mice and its proteome data could be applied to further $\mathrm{AD}$ researches, we performed a comparative analysis with existing $\mathrm{AD}$ proteome datasets. First, the proteome data of ADLP $^{\mathrm{APT}}$ mice were compared with that of human $\mathrm{AD}$ brain generated by Seyfried and Levey [50]. As a result, approximately $92 \%$ of the identified proteins were overlapped with the human $\mathrm{AD}$ proteome and approximately $30 \%$ of significantly regulated proteins in human $\mathrm{AD}$ data showed identical expression patterns with those of $\mathrm{ADLP}^{\mathrm{APT}}$ mice ( $p$-value $<0.05$ in both studies, respectively). We also assessed the commonality between our mouse model and the previously established 3xTg $\mathrm{AD}$ mouse model [73-75]. Notably, the comparative analysis indicated that our DEPs were largely different from those of other studies of 3xTg AD mice (data not shown). Most of our DEPs were neither detected nor showed consistent expression patterns in other studies. This may be attributed to the fact that the results of other studies were generated from 2-DIGE analysis [73] or the vesicular proteome isolated from the forebrain [74]. To the best of our knowledge, this study is the first to perform proteomic analysis in hippocampi developing both amyloid and NFT pathologies. These results indicate that the pathological relevance of our mouse model for $\mathrm{AD}$ research is valid at the protein level and in severe hippocampal pathologies.

\section{Conclusion}

We herein described a novel mouse model for $\mathrm{AD}$, namely ADLP ${ }^{\text {APT }}$ mouse. This model exhibited a rapid progression of $\mathrm{AD}$ pathology, including neuronal death and an accelerated NFT pathology that are particularly accelerated by the amyloid pathology. Thus, this model recapitulated the progression of human $\mathrm{AD}$. Using LCMS with 10-plex TMT isobaric labeling, we further constructed a hippocampal proteome database of ADLP mouse models. We used this database to understand the biological functions that were altered in each transgenic mouse model. This proteomic database also offers extensive resource of various molecular phenotypes in response to amyloid and NFT pathologies. Based on the bioinformatics analysis, we propose potential molecular targets for mechanistic studies and therapeutic development based on the proteomic database generated from our novel animal model.

\section{Additional files}

Additional file 1: Supplementary Methods. (DOCX $31 \mathrm{~kb}$ )

Additional file 2: Figure S1. Pathological characterization of a novel animal model of Alzheimer's disease. Figure S2. Activated neuroinflammation in a novel animal model of Alzheimer's disease. Figure S3. TMT-based protein quantification strategy. Figure S4. The comparative analysis between ADLPAPT and other AD proteome datasets. Figure S5. The quality assessment of MS analysis. Figure S6. The expression levels of exclusive DEPs ${ }^{\text {APT }}$ in other ADLP mice. Figure S7. Longitudinal expression changes of kinases involved in phosphorylation of tau protein. (PPTX $2370 \mathrm{~kb}$ )

Additional file 3: Table S1. All identified protein groups. (XLSX $1130 \mathrm{~kb}$ ) Additional file 4: Table S2. All quantified protein groups. (XLSX $4540 \mathrm{~kb}$ ) Additional file 5: Table S3. Significantly regulated proteins from t-test. (XLSX $170 \mathrm{~kb}$ )

Additional file 6: Table S4. DEPs from ANOVA test. (XLSX 391 kb) Additional file 7: Table S5. Canonical pathways enriched by IPA analysis. (XLSX $18 \mathrm{~kb}$ )

Additional file 8: Table S6. DEPs in ADLP ${ }^{A P T}$ mice, DEPS ${ }^{A P T}$. (XLSX 195 kb)

Additional file 9: Table S7. GO analysis results in ADLPAPT mice. (XLSX $41 \mathrm{~kb}$ )

Additional file 10: Table S8. IPA analysis results in ADLPAPT mice. (XLSX $53 \mathrm{~kb}$ )

Additional file 11: Table S9. Exclusive DEPs ${ }^{\mathrm{APT}}$. (XLSX $31 \mathrm{~kb}$ )

Additional file 12: Table S10. GO analysis of commonly significant proteins in human and ADLP ${ }^{\text {APT }}$. (XLSX 13 kb)

\section{Abbreviations}

ADLP: Alzheimer's disease-like pathology; APP: Amyloid precursor protein; Aß: Amyloid beta; BPRP: Basic pH reverse phase; DEPs: Differentially expressed proteins; GO: Gene ontology; IPA: Ingenuity pathway analysis; LCMS: Liquid chromatography - Mass spectrometry; LTP: Long term potentiation; NFT: Neurofibrillary tangle; PPI: Protein-protein interaction; TMT: Tandem mass tag

Acknowledgements

Not applicable.

Funding

This work was supported by the National Research Foundation grant (No. 20110030740 and 2017M3C9A5031597) to YK and NRF (2015R1A2A1A05001794, 2014M3C7A1046047, 2015M3C7A1028790, and MRC [2011-0030738]) to IM-J. DKK received a scholarship from the BK21-plus education program provided by the National Research Foundation of Korea.

Availability of data and materials

All data generated or analyzed during this study are included in this published article and its supplementary information files. The MS proteomics data in this paper have been deposited in the ProteomeXchange Consortium (http:// proteomecentral.proteomexchange.org) via the PRIDE partner repository [76]: dataset identifier PXD006214.

Username: reviewer22106@ebi.ac.uk, Password: eqd8pf32. 


\section{Authors' contributions}

DKK, JP, DH participated equally in the overall work. DKK, JP, DH, YK, and IM-J designed the research and experiments. DKK performed the immunohistochemistry, sarkosyl-insoluble tau fractionation, RT-PCR, and behavioral tests and wrote the manuscript. JP performed proteomics experiment and wrote the manuscript. DH contributed to the proteomics experiments and revised the manuscript. JY contributed to design sarkosyl-insoluble tau fractionation experiment and carry out the immunohistochemistry. AK contributed to do behavioral tests and prepare for biological experiments. JW contributed to analyze the MS-based proteomics data. YK and IM-J revised manuscript. All authors have read and approved the final manuscript.

\section{Ethics approval and consent to participate}

Animals were treated and maintained as per the Helsinki Treaty, the Principles of Laboratory Animal Care (NIH publication No. 85-23, revised 1985), and the Animal Care and Use Guidelines of Seoul National University, Seoul, Korea. All experimental procedures were reviewed and approved by the Institutional Animal Care and Use Committee (IACUC) of Seoul National University.

\section{Consent for publication}

Not applicable.

\section{Competing interests}

The authors declare that they have no competing interests.

\section{Publisher's Note}

Springer Nature remains neutral with regard to jurisdictional claims in published maps and institutional affiliations.

\section{Author details}

${ }^{1}$ Department of Biomedical Sciences, Seoul National University, College of Medicine, 103 Daehak-ro, Seoul 110-799, South Korea. ${ }^{2}$ Interdisciplinary Program in Bioengineering, College of Engineering, Seoul National University, 1 Gwanak-ro, Seoul 151-742, South Korea. ${ }^{3}$ Biomedical Research Institute, Seoul National University Hospital, 101 Daehak-ro, Seoul 110-744, South Korea. ${ }^{4}$ Neuroscience Research Institute, Seoul National University, College of Medicine, Seoul 110-799, South Korea.

Received: 1 August 2017 Accepted: 29 December 2017

Published online: 16 January 2018

\section{References}

1. Braak H, Braak E. Staging of Alzheimer's disease-related neurofibrillary changes. Neurobiol Aging. 1995;16:271-8. discussion 278-284

2. Hardy J, Selkoe DJ. The amyloid hypothesis of Alzheimer's disease: progress and problems on the road to therapeutics. Science. 2002;297:353-6.

3. Oddo S, Caccamo A, Shepherd JD, Murphy MP, Golde TE, Kayed R, Metherate R, Mattson MP, Akbari Y, LaFerla FM. Triple-transgenic model of Alzheimer's disease with plaques and tangles: intracellular Abeta and synaptic dysfunction. Neuron. 2003;39:409-21.

4. Oakley H, Cole SL, Logan S, Maus E, Shao P, Craft J, Guillozet-Bongaarts A, Ohno M, Disterhoft J, Van Eldik L, et al. Intraneuronal beta-amyloid aggregates, neurodegeneration, and neuron loss in transgenic mice with five familial Alzheimer's disease mutations: potential factors in amyloid plaque formation. J Neurosci. 2006;26:10129-40.

5. Lewis J, McGowan E, Rockwood J, Melrose H, Nacharaju P, Van Slegtenhorst M, Gwinn-Hardy K, Paul Murphy M, Baker M, Yu X, et al. Neurofibrillary tangles, amyotrophy and progressive motor disturbance in mice expressing mutant (P301L) tau protein. Nat Genet. 2000;25:402-5.

6. Bird CM, Burgess N. The hippocampus and memory: insights from spatial processing. Nat Rev Neurosci. 2008;9:182-94.

7. Aebersold R, Mann M. Mass-spectrometric exploration of proteome structure and function. Nature. 2016:537:347-55.

8. Choudhary C, Mann M. Decoding signalling networks by mass spectrometrybased proteomics. Nat Rev Mol Cell Biol. 2010;11:427-39.

9. Sharma K, Schmitt S, Bergner CG, Tyanova S, Kannaiyan N, Manrique-Hoyos N, Kongi K, Cantuti L, Hanisch UK, Philips MA, et al. Cell type- and brain region-resolved mouse brain proteome. Nat Neurosci. 2015;18:1819-31.

10. Zhang Y, Fonslow BR, Shan B, Baek MC, Yates JR 3rd. Protein analysis by shotgun/bottom-up proteomics. Chem Rev. 2013;113:2343-94.
11. Thompson A, Schafer J, Kuhn K, Kienle S, Schwarz J, Schmidt G, Neumann T, Johnstone R, Mohammed AK, Hamon C. Tandem mass tags: a nove quantification strategy for comparative analysis of complex protein mixtures by MS/MS. Anal Chem. 2003;75:1895-904.

12. McAlister GC, Huttlin EL, Haas W, Ting L, Jedrychowski MP, Rogers JC, Kuhn K, Pike I, Grothe RA, Blethrow JD, Gygi SP. Increasing the multiplexing capacity of TMTs using reporter ion isotopologues with isobaric masses. Anal Chem. 2012;84:7469-78.

13. Christoforou A, Mulvey CM, Breckels LM, Geladaki A, Hurrell T, Hayward PC, Naake T, Gatto L, Viner R, Martinez Arias A, Lilley KSA. Draft map of the mouse pluripotent stem cell spatial proteome. Nat Commun. 2016;7:8992.

14. Murphy JP, Stepanova E, Everley RA, Paulo JA, Gygi SP. Comprehensive temporal protein dynamics during the Diauxic shift in Saccharomyces Cerevisiae. Mol Cell Proteomics. 2015;14:2454-65.

15. Weekes MP, Tomasec P, Huttlin EL, Fielding CA, Nusinow D, Stanton RJ, Wang EC, Aicheler R, Murrell I, Wilkinson GW, et al. Quantitative temporal viromics: an approach to investigate host-pathogen interaction. Cell. 2014;157:1460-72.

16. Chick JM, Munger SC, Simecek P, Huttlin EL, Choi K, Gatti DM, Raghupathy $\mathrm{N}$, Svenson KL, Churchill GA, Gygi SP. Defining the consequences of genetic variation on a proteome-wide scale. Nature. 2016;534:500-5.

17. Gotz J, Ittner LM. Animal models of Alzheimer's disease and frontotemporal dementia. Nat Rev Neurosci. 2008;9:532-44.

18. Han D, Moon S, Kim Y, Kim J, Jin J, Kim Y. In-depth proteomic analysis of mouse microglia using a combination of FASP and StageTip-based, high pH, reversed-phase fractionation. Proteomics. 2013;13:2984-8.

19. Han D, Jin J, Woo J, Min H, Kim Y. Proteomic analysis of mouse astrocytes and their secretome by a combination of FASP and StageTip-based, high pH, reversed-phase fractionation. Proteomics. 2014;14:1604-9.

20. Kulak NA, Pichler G, Paron I, Nagaraj N, Mann M. Minimal, encapsulated proteomic-sample processing applied to copy-number estimation in eukaryotic cells. Nat Methods. 2014;11:319-24

21. Tyanova S, Temu T, Sinitcyn P, Carlson A, Hein MY, Geiger T, Mann M, Cox J. The Perseus computational platform for comprehensive analysis of (prote)omics data. Nat Methods. 2016:13:731-40.

22. Benjamini $Y$, Hochberg Y. Controlling the false discovery rate - a practical and powerful approach to multiple testing. J Roy Stat Soc B Met. 1995:57:289-300.

23. Huang d W, Sherman BT, Lempicki RA. Systematic and integrative analysis of large gene lists using DAVID bioinformatics resources. Nat Protoc. 2009:4:44-57.

24. Kramer A, Green J, Pollard J Jr, Tugendreich S. Causal analysis approaches in ingenuity pathway analysis. Bioinformatics. 2014;30:523-30.

25. Szklarczyk D, Franceschini A, Wyder S, Forslund K, Heller D, Huerta-Cepas J, Simonovic M, Roth A, Santos A, Tsafou KP, et al. STRING v10: protein-protein interaction networks, integrated over the tree of life. Nucleic Acids Res. 2015;43:D447-52.

26. Shannon P, Markiel A, Ozier O, Baliga NS, Wang JT, Ramage D, Amin N, Schwikowski B, Ideker T. Cytoscape: a software environment for integrated models of biomolecular interaction networks. Genome Res. 2003;13:2498-504.

27. Wong PC, Cai H, Borchelt DR, Price DL. Genetically engineered mouse models of neurodegenerative diseases. Nat Neurosci. 2002:5:633-9.

28. Wisniewski JR, Zougman A, Nagaraj N, Mann M. Universal sample preparation method for proteome analysis. Nat Methods. 2009:6:359-62.

29. Verghese PB, Castellano JM, Holtzman DM, Apolipoprotein E. In Alzheimer's disease and other neurological disorders. Lancet Neurol. 2011;10:241-52.

30. JT Y, Tan $L$. The role of clusterin in Alzheimer's disease: pathways, pathogenesis, and therapy. Mol Neurobiol. 2012;45:314-26.

31. Brouwers N, Bettens K, Gijselinck I, Engelborghs S, Pickut BA, Van Miegroet $H$, Montoya AG, Mattheijssens M, Peeters K, De Deyn PP, et al. Contribution of TARDBP to Alzheimer's disease genetic etiology. J Alzheimers Dis. 2010;21:423-30.

32. Shibata N, Ohnuma T, Baba H, Arai H. Genetic association analysis between TDP-43 polymorphisms and Alzheimer's disease in a Japanese population. Dement Geriatr Cogn Disord. 2009:28:325-9.

33. Schjeide BM, Hooli B, Parkinson M, Hogan MF, DiVito J, Mullin K, Blacker D, Tanzi RE, Bertram L. GAB2 as an Alzheimer disease susceptibility gene: follow-up of genomewide association results. Arch Neurol. 2009:66:250-4.

34. Feulner TM, Laws SM, Friedrich P, Wagenpfeil S, Wurst SH, Riehle C, Kuhn KA, Krawczak M, Schreiber S, Nikolaus S, et al. Examination of the current top candidate genes for AD in a genome-wide association study. Mol Psychiatry. 2010;15:756-66.

35. Sleegers K, Bettens K, Brouwers N, Engelborghs S, van Miegroet H, De Deyn PP, Van Broeckhoven C. Common variation in GRB-associated binding protein 2 (GAB2) and increased risk for Alzheimer dementia. Hum Mutat. 2009;30:E338-44. 
36. Rayaprolu S, Mullen B, Baker M, Lynch T, Finger E, Seeley WW, Hatanpaa KJ, Lomen-Hoerth C, Kertesz A, Bigio EH, et al. TREM2 in neurodegeneration: evidence for association of the $\mathrm{p} . \mathrm{R} 47 \mathrm{H}$ variant with frontotemporal dementia and Parkinson's disease. Mol Neurodegener. 2013:8:19.

37. Kim WS, Weickert CS, Garner B. Role of ATP-binding cassette transporters in brain lipid transport and neurological disease. J Neurochem. 2008;104:1145-66.

38. Naj AC, Jun G, Beecham GW, Wang LS, Vardarajan BN, Buros J, Gallins PJ, Buxbaum JD, Jarvik GP, Crane PK, et al. Common variants at MS4A4/ MS4A6E, CD2AP, CD33 and EPHA1 are associated with late-onset Alzheimer's disease. Nat Genet. 2011:43:436-41.

39. Hol EM, Roelofs RF, Moraal E, Sonnemans MA, Sluijs JA, Proper EA, de Graan PN, Fischer DF, van Leeuwen FW. Neuronal expression of GFAP in patients with Alzheimer pathology and identification of novel GFAP splice forms. Mol Psychiatry. 2003;8:786-96.

40. Potkin SG, Guffanti G, Lakatos A, Turner JA, Kruggel F, Fallon JH, Saykin AJ, Orro A, Lupoli S, Salvi E, et al. Hippocampal atrophy as a quantitative trait in a genome-wide association study identifying novel susceptibility genes for Alzheimer's disease. PLoS One. 2009;4:e6501.

41. Grupe A, Abraham R, Li Y, Rowland C, Hollingworth P, Morgan A, Jehu L, Segurado R, Stone D, Schadt E, et al. Evidence for novel susceptibility genes for late-onset Alzheimer's disease from a genome-wide association study of putative functional variants. Hum Mol Genet. 2007;16:865-73.

42. Zorzetto M, Datturi F, Divizia L, Pistono C, Campo I, De Silvestri A, Cuccia M, Ricevuti G. Complement C4A and C4B gene copy number study in Alzheimer's disease patients. Curr Alzheimer Res. 2017;14:303-8.

43. Tarawneh R, D'Angelo G, Crimmins D, Herries E, Griest T, Fagan AM, Zipfel GJ, Ladenson JH, Morris JC, Holtzman DM. Diagnostic and prognostic utility of the synaptic marker Neurogranin in Alzheimer disease. JAMA Neurol. 2016;73:561-71.

44. Lee JH, Cheng R, Barral S, Reitz C, Medrano M, Lantigua R, JimenezVelazquez IZ, Rogaeva E. St George-Hyslop PH, Mayeux R. Identification of novel loci for Alzheimer disease and replication of CLU, PICALM, and BIN1 in Caribbean Hispanic individuals. Arch Neurol. 2011;68:320-8.

45. Jayadev S, Leverenz JB, Steinbart E, Stahl J, Klunk W, CE Y, Bird TD. Alzheimer's disease phenotypes and genotypes associated with mutations in presenilin 2. Brain. 2010;133:1143-54.

46. Brouwers N, Sleegers K, Engelborghs S, Maurer-Stroh S, Gijselinck I, van der Zee J, Pickut BA, Van den Broeck M, Mattheijssens M, Peeters K, et al. Genetic variability in progranulin contributes to risk for clinically diagnosed Alzheimer disease. Neurology. 2008;71:656-64.

47. Zhao $Y$, Ho P, Yih Y, Chen C, Lee WL, Tan EK. LRRK2 variant associated with Alzheimer's disease. Neurobiol Aging. 2011;32:1990-3.

48. Ersing I, Nobre L, Wang LW, Soday L, Ma Y, Paulo JA, Narita Y, Ashbaugh CW, Jiang C, Grayson NE, et al. A temporal proteomic map of Epstein-Barr virus Lytic replication in B cells. Cell Rep. 19:1479-93.

49. Hughes CS, McConechy MK, Cochrane DR, Nazeran T, Karnezis AN, Huntsman DG, Morin GB. Quantitative profiling of single formalin fixed tumour sections: proteomics for translational research. Sci Rep. 2016;6:34949.

50. Seyfried NT, Dammer EB, Swarup V, Nandakumar D, Duong DM, Yin L, Deng Q, Nguyen T, Hales CM, Wingo T, et al. A multi-network approach identifies protein-specific co-expression in asymptomatic and symptomatic Alzheimer's disease. Cell Syst. 2017;4:60-72 e64.

51. Karran E, Mercken M, De Strooper B. The amyloid cascade hypothesis for Alzheimer's disease: an appraisal for the development of therapeutics. Nat Rev Drug Discov. 2011;10:698-712.

52. Martin L, Latypova X, Wilson CM, Magnaudeix A, Perrin ML, Yardin C, Terro F. Tau protein kinases: involvement in Alzheimer's disease. Ageing Res Rev. 2013;12:289-309.

53. Takashima A, Noguchi K, Sato K, Hoshino T, Imahori K. Tau protein kinase I is essential for amyloid beta-protein-induced neurotoxicity. Proc Natl Acad Sci U S A. 1993;90:7789-93

54. Li G, Yin H, Kuret J. Casein kinase 1 delta phosphorylates tau and disrupts its binding to microtubules. J Biol Chem. 2004;279:15938-45.

55. Wagner U, Utton M, Gallo JM, Miller CC. Cellular phosphorylation of tau by GSK-3 beta influences tau binding to microtubules and microtubule organisation. J Cell Sci. 1996;109(Pt 6):1537-43.

56. Virdee K, Yoshida H, Peak-Chew S, Goedert M. Phosphorylation of human microtubule-associated protein tau by protein kinases of the AGC subfamily. FEBS Lett. 2007;581:2657-62.

57. Pooler AM, Polydoro M, Maury EA, Nicholls SB, Reddy SM, Wegmann S, William C, Saqran L, Cagsal-Getkin O, Pitstick R, et al. Amyloid accelerates tau propagation and toxicity in a model of early Alzheimer's disease. Acta Neuropathol Commun. 2015;3:14.

58. Tseng JH, Xie L, Song S, Xie Y, Allen L, Ajit D, Hong JS, Chen X, Meeker RB, Cohen TJ. The Deacetylase HDAC6 mediates endogenous Neuritic tau pathology. Cell Rep. 2017;20:2169-83.

59. Kook SY, Seok Hong H, Moon M, Mook-Jung I. Disruption of blood-brain barrier in Alzheimer disease pathogenesis. Tissue Barriers. 2013;1:e23993.

60. Marques F, Sousa JC, Sousa N, Palha JA. Blood-brain-barriers in aging and in Alzheimer's disease. Mol Neurodegener. 2013;8:38

61. Laurent C, Dorothee $G$, Hunot S, Martin E, Monnet $Y$, Duchamp M, Dong $Y$, Legeron FP, Leboucher A, Burnouf S, et al. Hippocampal T cell infiltration promotes neuroinflammation and cognitive decline in a mouse model of tauopathy. Brain. 2017:140:184-200.

62. Baik SH, Cha MY, Hyun YM, Cho H, Hamza B, Kim DK, Han SH, Choi H, Kim $\mathrm{KH}$, Moon $\mathrm{M}$, et al. Migration of neutrophils targeting amyloid plaques in Alzheimer's disease mouse model. Neurobiol Aging. 2014;35:1286-92.

63. Zenaro E, Pietronigro E, Della Bianca V, Piacentino G, Marongiu L, Budui S, Turano E, Rossi B, Angiari S, Dusi S, et al. Neutrophils promote Alzheimer's disease-like pathology and cognitive decline via LFA-1 integrin. Nat Med. 2015;21:880-6.

64. Hohsfield LA, Humpel C. Migration of blood cells to beta-amyloid plaques in Alzheimer's disease. Exp Gerontol. 2015;65:8-15.

65. Chesser AS, Pritchard SM, Johnson GV. Tau clearance mechanisms and their possible role in the pathogenesis of Alzheimer disease. Front Neurol. 2013;4:122.

66. Kim Y, Kim C, Son SM, Song H, Hong HS, Han SH, Mook-Jung I. The novel RAGE interactor PRAK is associated with autophagy signaling in Alzheimer's disease pathogenesis. Mol Neurodegener. 2016;11:4.

67. Saura CA, Valero J. The role of CREB signaling in Alzheimer's disease and other cognitive disorders. Rev Neurosci. 2011;22:153-69.

68. Thomas GM, Huganir RL. MAPK cascade signalling and synaptic plasticity. Nat Rev Neurosci. 2004:5:173-83.

69. LaFerla FM, Oddo S. Alzheimer's disease: Abeta, tau and synaptic dysfunction. Trends Mol Med. 2005;11:170-6.

70. Zhu Y, Hou H, Rezai-Zadeh K, Giunta B, Ruscin A, Gemma C, Jin J, Dragicevic N, Bradshaw P, Rasool S, et al. CD45 deficiency drives Amyloid- $\beta$ peptide Oligomers and neuronal loss in Alzheimer's disease mice. J Neurosci. 2011:31:1355-65.

71. ElAli A, Rivest $\mathrm{S}$. The role of $A B C B 1$ and $A B C A 1$ in beta-amyloid clearance at the neurovascular unit in Alzheimer's disease. Front Physiol. 2013:4:45.

72. Latasiewicz J, Artz A, Jing D, Blanco MP, Currie SM, Avila MV, Schnoor M, Vestweber D. HS1 deficiency impairs neutrophil recruitment in vivo and activation of the small GTPases Rac1 and Rap1. J Leukoc Biol. 2017;101: $1133-1142$.

73. Ciavardelli D, Silvestri E, Del Viscovo A, Bomba M, De Gregorio D, Moreno M, Di llio C, Goglia F, Canzoniero LM, Sensi SL. Alterations of brain and cerebellar proteomes linked to Abeta and tau pathology in a female tripletransgenic murine model of Alzheimer's disease. Cell Death Dis. 2010;1:e90.

74. Rhein V, Song X, Wiesner A, Ittner LM, Baysang G, Meier F, Ozmen L, Bluethmann H, Drose S, Brandt $U$, et al. Amyloid-beta and tau synergistically impair the oxidative phosphorylation system in triple transgenic Alzheimer's disease mice. Proc Natl Acad Sci U S A. 2009;106:20057-62.

75. Martin B, Brenneman R, Becker KG, Gucek M, Cole RN, Maudsley S. iTRAQ analysis of complex proteome alterations in 3xTgAD Alzheimer's mice: understanding the interface between physiology and disease. PLoS One. 2008;3:e2750.

76. Vizcaino JA, Deutsch EW, Wang R, Csordas A, Reisinger F, Rios D, Dianes JA, Sun Z, Farrah T, Bandeira N, et al. ProteomeXchange provides globally coordinated proteomics data submission and dissemination. Nat Biotechnol. 2014;32:223-6.

\section{Submit your next manuscript to BioMed Central and we will help you at every step:}

- We accept pre-submission inquiries

- Our selector tool helps you to find the most relevant journal

- We provide round the clock customer support

- Convenient online submission

- Thorough peer review

- Inclusion in PubMed and all major indexing services

- Maximum visibility for your research

Submit your manuscript at www.biomedcentral.com/submit 